\title{
MATRIX-VALUED NEVANLINNA-PICK INTERPOLATION WITH COMPLEXITY CONSTRAINT: AN OPTIMIZATION APPROACH*
}

\author{
ANDERS BLOMQVIST $\dagger$, ANDERS LINDQUIST $\dagger$, AND RYOZO NAGAMUNE $\dagger$
}

\begin{abstract}
Over the last several years a new theory of Nevanlinna-Pick interpolation with complexity constraint has been developed for scalar interpolants. In this paper we generalize this theory to the matrix-valued case, also allowing for multiple interpolation points. We parameterize a class of interpolants consisting of "most interpolants" of no higher degree than the central solution in terms of spectral zeros. This is a complete parameterization, and for each choice of interpolant we provide a convex optimization problem for determining it. This is derived in the context of duality theory of mathematical programming. To solve the convex optimization problem, we employ a homotopy continuation technique previously developed for the scalar case. These results can be applied to many classes of engineering problems, and, to illustrate this, we provide some examples. In particular, we apply our method to a benchmark problem in multivariate robust control. By constructing a controller satisfying all design specifications but having only half the McMillan degree of conventional $H^{\infty}$ controllers, we demonstrate the efficiency of our method.
\end{abstract}

\section{INTRODUCTION}

Applications of Nevanlinna-Pick interpolation abound in robust control [15, 21, 23, $28,32,33,46,47]$, signal processing $[4,5,6,9,25,31]$ and maximal power transfer [50] in circuit theory, to mention a few. Since the interpolant has a specific interpretation as a transfer function in all these applications, it is important to restrict its degree, and at the same time satisfy all design specifications. The lack of insight provided by the classical techniques of Nevanlinna-Pick interpolation into questions regarding the degree of various interpolants has therefore been a limiting factor in this approach. In fact, the designer has generally been confined to the so called central solution, or the essentially equivalent solution derived by Nehari approach, the only type of solution for which algorithms have been available.

Over the last several years a new theory of analytic interpolation with complexity constraint has been developed for scalar interpolants $[8,7,12,9,11,5]$. The basic idea is to parameterize complete classes of interpolants of at most a given degree in a smooth fashion, providing tuning parameters for modifying the design without increasing the complexity. This is done in the context of duality theory of mathematical programming, providing convex optimization problems for determining any interpolant belonging to such a class. In this context, new paradigms for spectral estimation [6, 4, 5] and robust controller synthesis [7, 38, 39, 40, 41, 42] have been developed in the scalar-input/scalar-output case.

* This research was supported by grants from TFR and the Swedish Research Council.

$\dagger$ Division of Optimization and Systems Theory, Department of Mathematics, Royal Institute of Technology, SE 10044 Stockholm, Sweden. 
However, all these results are for scalar interpolants, while the multivariable case is clearly more interesting and important in most of the applications mentioned above. For example, while our design procedures in robust control [7, 38, 39, 40, 41, 42] compare very favorably to $H^{\infty}$ control methods in the scalar case, it is in the multivariable case that they have a chance to outperform classical control methods in general.

Motivated by this, in this paper we generalize the theory of [7] to the matrixvalued case, also allowing for multiple interpolation points. We parameterize a class of interpolants consisting of "most interpolants" of no higher degree than the central solution in terms of spectral zeros. This is a complete parameterization, and for each choice of interpolant we provide a convex optimization problem for determining it. This is derived in the context of a duality theory, generalizing that of $[7,8]$; also see the survey in [9]. To do this, we regard the Nevanlinna-Pick interpolation as a generalized moment problem, to proceed along the lines of $[11,10]$. However, the present multivariate case introduces new nontrivial and challenging issues.

The outline of this paper is as follows. In Section 2 we provide some motivation examples, introducing the reader to matrix interpolation in the context of signal processing and control. Section 3 is a preliminary in which we formulate the matrixvalued interpolation problem, first defining a corresponding class of rational strictly positive real functions with complexity constraint. We reformulate the problem as a generalized moment problem and provide a necessary and sufficient condition for existence of solutions, which we then interpret as a generalized Pick condition. The main theorems are presented in Section 4 and proved in Section 5. Generalizing results in $[8,7,9,11,10]$ to matrix-valued analytic interpolation theory, we present a smooth, complete parameterization of the set of matrix-valued interpolants with complexity constraint in the context of duality theory of mathematical programming. In fact, to each choice of parameters, there is a pair of dual optimization problems, the optima of which uniquely determine the interpolant. The primal problem amounts to maximizing a generalized entropy gain subject to the interpolation conditions, while the dual problem is a convex optimization problem with a unique minimum. In Section 6, an algorithm for solving the dual problem is provided. Here we generalize to the matrix setting an approach first applied to the covariance extension problem in [22] and then extended in $[40,2]$ to Nevanlinna-Pick interpolation. Since the dual problem is illbehaved close to the boundary, we reformulate the optimization problem to eliminate this property. This is done at the expense of global convexity, but the new functional is still locally strictly convex in a neighborhood of a unique minimizing point so that we can solve the problem by a homotopy continuation method. In Section 7 , finally, a numerical example in robust control is presented. We consider a popular benchmark problem and show that our design achieves the design specifications with a controller of much lower degree than that of the $H_{\infty}$ design with weighting functions.

\section{Motivating EXAMPles From Signal PROCESSing AND CONTROL}

To justify the problem formulation of this paper, we begin by briefly considering some motivating examples.

Example 2.1. Suppose we are given a sequence $C_{0}, C_{1}, \ldots, C_{n}$ of matrix-valued covariance lags

$$
C_{k}=\mathrm{E}\left\{y_{t+k} y_{t}^{*}\right\}, \quad k=0,1, \ldots, n
$$


of some real $\ell$-dimensional stationary stochastic process $\left\{y_{t} ; t \in \mathbb{Z}\right\}$ with the property that the block Toeplitz matrix

$$
\left[\begin{array}{cccc}
C_{0} & C_{1}^{\top} & \cdots & C_{n}^{\top} \\
C_{1} & C_{0} & \cdots & C_{n-1}^{\top} \\
\vdots & \vdots & \ddots & \vdots \\
C_{n} & C_{n-1} & \cdots & C_{0}
\end{array}\right]
$$

is positive definite. Such covariance lags can be determined from observations of $\left\{y_{t}\right\}$ via an ergodic estimate (see, e.g., [45]). The problem is to estimate the spectral density $\Phi\left(e^{i \theta}\right), \theta \in[-\pi, \pi]$ of $\left\{y_{t}\right\}$ by matching the given covariance sequence:

$$
\frac{1}{2 \pi} \int_{-\pi}^{\pi} e^{i k \theta} \Phi\left(e^{i \theta}\right) d \theta=C_{k}, \quad k=0,1, \ldots, n .
$$

Often one is more interested in the minimum-phase ${ }^{1}$ spectral factor of $\Phi$, i.e., a solution $V$ of

$$
V(z) V\left(z^{-1}\right)^{\top}=\Phi(z)
$$

with all its poles and zeros in the complement of the closed unit disc. In fact, $V$ represents a filter that shapes white noise into a process $\left\{y_{t}\right\}$ with the spectral density $\Phi$.

The problem of determining a $\Phi(z)$ that is positive on the unit circle and satisfies the finite number of moment equations (2.1) has infinitely many solution. However, for design purposes, we are interested in solutions that are rational of reasonably low degree. A favorite solution is the one that maximizes the entropy gain

$$
\frac{1}{2 \pi} \int_{-\pi}^{\pi} \log \operatorname{det} \Phi\left(e^{i \theta}\right) d \theta
$$

The shaping filter $V$ corresponding to this maximum-entropy solution has the form

$$
V(z)=z^{n} R(z)^{-1}
$$

where $R(z)$ is a matrix polynomial of degree $n$ whose coefficients are the unique solution of the normal equations, which are linear and can be solved by means of a matrix-version of the Levinson algorithm [45]; for some earlier papers, see [48, 49, 36]. Clearly this $V$ has degree $n \ell$ and has $n$ zeros all located at the origin.

As a first step toward generalizing this, one might ask whether there is a solution of the form

$$
V(z)=\rho(z) R(z)^{-1}
$$

where $\rho(z)$ is an arbitrary scalar stable polynomial of degree $n$. This is a matrix version of a question answered in the affirmative in [25], the question of uniqueness left open and finally settled in [13]. In this paper, we shall prove that, for each $\rho(z)$,

\footnotetext{
${ }^{1}$ This is a somewhat nonstandard use of the term minimum-phase caused by having the the unit disc as the region of analyticity. From a mathematical point of view, the term outer might be more appropriate.
} 


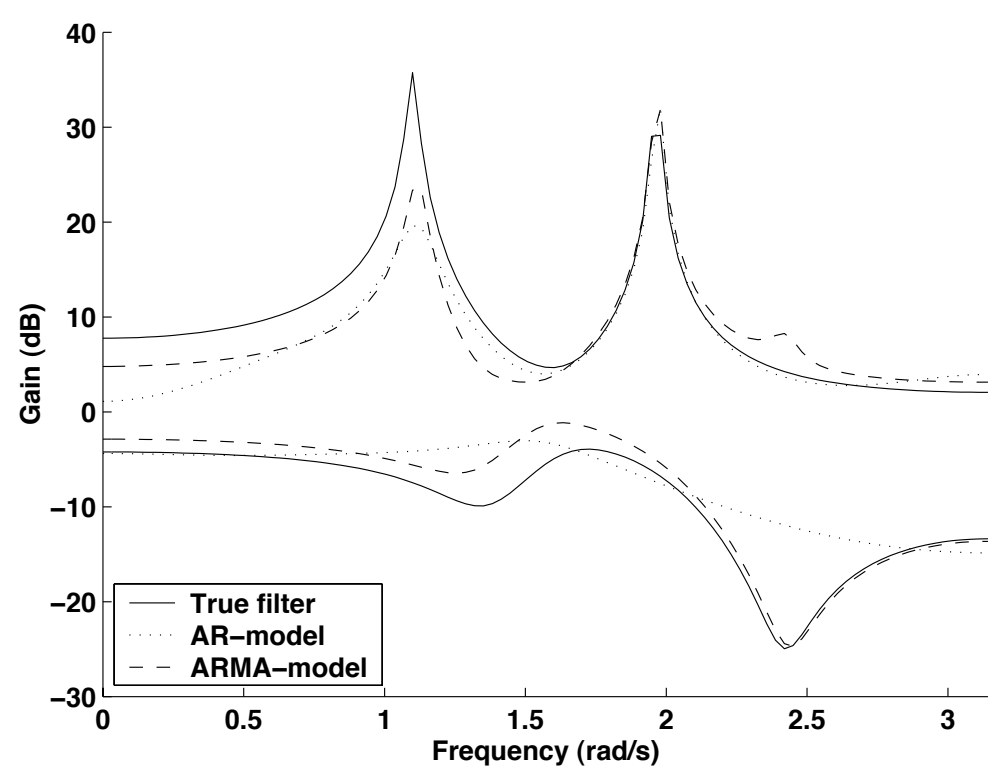

Figure 1: Various spectral estimates compared to the true spectral density.

there is one and only one $R(z)$ so that $\Phi$ defined by (2.2) and (2.3) satisfies the moment conditions (2.1), and it is the $\Phi$ maximizing the generalized entropy gain

$$
\frac{1}{2 \pi} \int_{-\pi}^{\pi}\left|\rho\left(e^{i \theta}\right)\right|^{2} \log \operatorname{det} \Phi\left(e^{i \theta}\right) d \theta .
$$

This generalizes the corresponding scalar result in [8]. We shall also prove that this parameterization is smooth, forming a family of covariance extensions having a complexity no higher than the maximum entropy solution.

This spectral estimation problem can also be formulated as a matrix NevanlinnaPick problem. In fact, as we shall see in Section 3 , a strictly positive real $\ell \times \ell$ matrix function $F$ satisfies the interpolation condition

$$
F(0)=\frac{1}{2} C_{0}, \quad \frac{1}{k !} F^{(k)}(0)=C_{k}, \quad k=1,2, \ldots, n,
$$

if and only if the spectral density

$$
\Phi(z)=F(z)+F\left(z^{-1}\right)^{\top}
$$

satisfies (2.1).

As an example, consider a two-dimensional stationary stochastic process generated by passing white noise through a known shaping filter. Observing a sample sequence of this process, we want to recover the true shaping filter from a finite window $C_{0}, C_{1}, \ldots, C_{n}$ of $2 \times 2$ covariance lags obtained from this observed data via ergodic estimates, while restricting the model order. The singular values of the estimated spectral densities for two different solutions are plotted in Figure 1, together with those of the true spectral density. The maximum-entropy solution, i.e., the ARmodel determined by the matrix-version of the Levinson algorithm is depicted with a dotted line. By choosing the tuning-parameter polynomial $\rho(z)$ appropriately, we obtain instead the ARMA model, depicted with a dashed line. Note that this method also works for generic data. Hence, the existence of a "true model" is not required. 
Example 2.2. Let $\mathrm{P}$ be a linear control system with a vector-valued input $u$ and a vector-valued output $y$, having a rational transfer function $P(s)$ with unstable poles and non-minimum-phase zeros; these are the poles and zeros, including multiplicities, of $P(s)$ that are located in the right half plane $\{z: \operatorname{Re} z \geq 0\}$. We want to design a compensator $\mathrm{C}$ of low complexity so that the closed-loop system depicted in Figure 1 is internally stable, attenuates the effect of the disturbance $d$, and tracks the reference signal $r$.

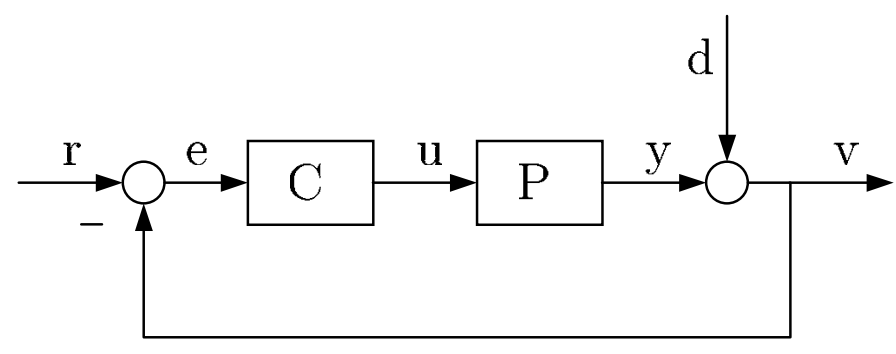

Figure 2: A feedback system.

This problem is standard in the robust control literature (see, e.g., [23, 21, 51]). Internal stability is achieved by requiring that the sensitivity function, i.e., the closedloop transfer function

$$
S(s)=[I+P(s) C(s)]^{-1}
$$

from the disturbance $d$ to the output $v$, is stable (all poles in the open left half plane) and satisfies an interpolation condition. Substituting the Youla-parameterization into (2.4) yields a model matching form:

$$
S(s)=T_{1}(s)-T_{2}(s) Q(s) T_{3}(s),
$$

where $T_{j}, j=1,2,3$ and $Q$ are stable rational matrices with $Q$ arbitrary. By the procedure of [16], we can transform this equation into

$$
\tilde{S}(s)=\tilde{T}_{1}(s)-\phi(s) Q(s), \quad\|S\|_{\infty}=\|\tilde{S}\|_{\infty},
$$

where $\phi$ is a scalar inner function having zeros at the unstable poles and zeros of $\mathrm{P}$, and $\tilde{T}_{1}$ can be determined from $T_{j}, j=1,2,3$. If these poles and zeros, denoted by $s_{0}, s_{1}, \ldots, s_{n}$, are distinct, the interpolation condition becomes

$$
\tilde{S}\left(s_{k}\right)=\tilde{T}_{1}\left(s_{k}\right), \quad k=0,1, \ldots, n,
$$

whereas any multiple point has to be handled in a separate way. If $s_{k}$ is an interpolation point of multiplicity $\nu$ so that $s_{k}=s_{k+1}=\cdots=s_{k+\nu-1}$, then the equations in (2.7) corresponding to $s_{k+1}=\cdots=s_{k+\nu-1}$ are replaced by

$$
\tilde{S}^{(j)}\left(s_{k}\right)=\tilde{T}_{1}^{(j)}\left(s_{k}\right), j=1, \ldots, \nu-1 .
$$

If $P(s)$ is strictly proper, we also need to add interpolation conditions at infinity to ensure that the controller is proper. If the first Markov parameter is nonsingular, we only need one condition, namely $S(\infty)=I$, whereas the interpolation conditions $S^{\prime}(\infty)=\cdots=S^{(r-1)}(\infty)=0$ need to be added if $P(s)$ has relative degree $r$. 
Disturbance attenuation and reference tracking are achieved by bounding the $H^{\infty}$ norm of the sensitivity function, i.e.,

$$
\|S\|_{\infty}=\|\tilde{S}\|_{\infty}<\gamma
$$

The lowest such bound, i.e., the infimum of $\|\tilde{S}\|_{\infty}$ over all stable $\tilde{S}$ satisfying (2.7) and (2.8), will be denoted by $\gamma_{\text {opt }}$. There are optimal solutions achieving this bound, and their largest singular values are uniform over the spectrum. However, in general one would like to shape the sensitivity function to obtain low sensitivity in designated part of the spectrum, which, due to the water-bed effect [44], is done at the expense of higher sensitivity in some other part of the spectrum. To achieve this, it is customary to use weighting functions, which however could increase the degree of the sensitivity function considerably, and hence the compensator.

However, we prefer sensitivity functions of low complexity, and therefore we would like to avoid weighting functions. To this end and to allow for greater design flexibility, we consider suboptimal solutions, of which there are infinitely many. Given some $\gamma>\gamma_{\text {opt }}$, we consider the whole class of stable $\tilde{S}$ satisfying $(2.7)-(2.9)$ and some complexity constraint. In this class we would like to choose the one that best satisfies the additional specifications of sensitivity shaping. In this paper, we shall give a smooth, complete parameterization of such a class.

To bring this problem in conformity with the problem formulation in Section 3, we transform first the interpolation points $s_{0}, s_{1}, \ldots, s_{n}$ in the right half plane to $z_{0}, z_{1}, \ldots, z_{n}$ in the unit circle, via the linear fractional transformation $z_{k}=(1-$ $\left.s_{k}\right)\left(1+s_{k}\right)^{-1}$, and then the function $\tilde{S}$ to

$$
F(z):=\left[\gamma I-\tilde{S}\left(\frac{1-z}{1+z}\right)\right]\left[\gamma I+\tilde{S}\left(\frac{1-z}{1+z}\right)\right]^{-1}
$$

For each $\tilde{S}$ satisfying (2.9), the new function $F$ is analytic in the unit disc and has the property that $F\left(e^{i \theta}\right)+F\left(e^{-i \theta}\right)^{\top}>0$ for all $\theta$. Let us call such a function a (matrixvalued) Carathéodory function. The problem is then reduced to finding a rational Carathéodory function $F$ that has low complexity and satisfies the corresponding interpolation condition

$$
F\left(z_{k}\right)=W_{k}
$$

for each $k$ such that $z_{k}$ has multiplicity one and

$$
\frac{1}{j !} F^{(j)}\left(z_{k}\right)=W_{k+j}, \quad j=0,1, \ldots, \nu-1
$$

whenever $z_{k}$ has multiplicity $\nu$ and $z_{k}=z_{k+1}=\cdots=z_{k+\nu-1}$. It is straightforward, but tedious in the multiple-point case, to determine the interpolation values $W_{0}, W_{1}, \ldots, W_{n}$ from $(2.7)$ and (2.8).

To illustrate the design flexibility of our approach, we consider an example in control, namely the double inverted pendulum depicted in Figure 3. The linearized model 
for $m=1(\mathrm{~kg})$ and $l=1(\mathrm{~m})$ is given in [17, p. 37] as

$$
\begin{aligned}
& \dot{x}=\left[\begin{array}{rrrr}
0 & 1 & 0 & 0 \\
g & 0 & -g & 0 \\
0 & 0 & 0 & 1 \\
-g & 0 & 3 g & 0
\end{array}\right] x+\left[\begin{array}{rr}
0 & 0 \\
1 & -2 \\
0 & 0 \\
-2 & 5
\end{array}\right]\left[\begin{array}{l}
u_{1} \\
u_{2}
\end{array}\right], \\
& y=\left[\begin{array}{llll}
1 & 0 & 0 & 0 \\
0 & 0 & 1 & 0
\end{array}\right] x
\end{aligned}
$$

where $x:=\left[\begin{array}{llll}\theta_{1} & \dot{\theta}_{1} & \theta_{2} & \dot{\theta}_{2}\end{array}\right]^{\top}$ is the state. This is our plant $\mathrm{P}$. The goal is to design a stabilizing controller $\mathrm{C}$ which is robust against constant disturbances $d$ and zero reference signal $(r=0)$. The plant transfer function has two unstable real poles and is of relative degree two, thus yielding four interpolation conditions.

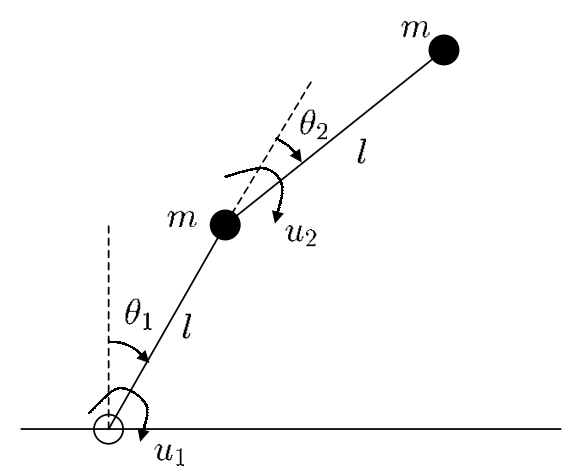

Figure 3: A double inverted pendulum.

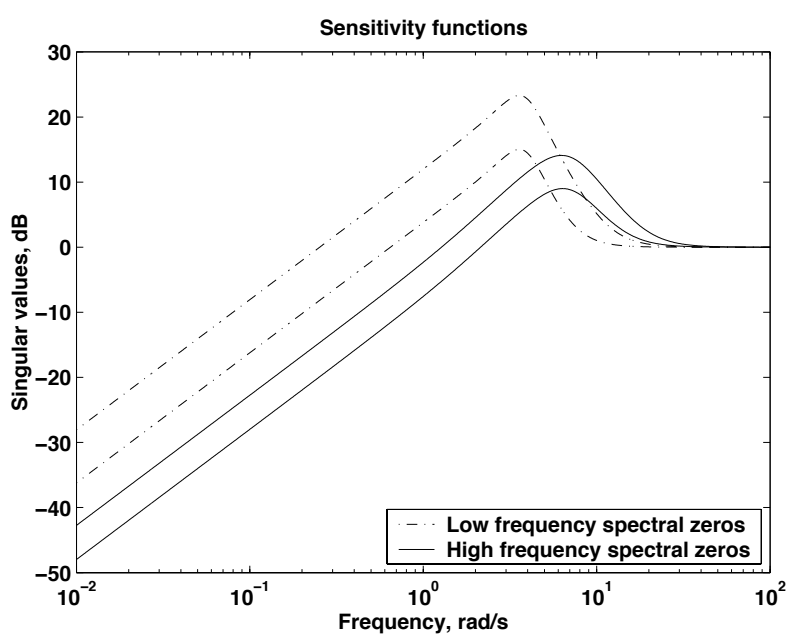

Figure 4: Frequency responses for various tunings of $S$.

Using the methods of this paper, we can now compute an arbitrary strictly proper controller in a class of controllers of degree at most eight, satisfying the specifications, by choosing the tuning parameters appropriately. Figure 4 shows the (singularvalue) frequency responses of two sensitivity functions in this class. One, plotted with 
dashed-dotted lines, gives a small bandwidth but large robustness against noise at the output, whereas the other, plotted with solid lines, provides a large bandwidth and lower peak gain but a small robustness to noise. Therefore, using the methods of this paper, the controller with the appropriate frequency response can be determined by tuning certain design parameters to satisfy the specifications.

\section{THE INTERPOLATION PROBLEM}

To formulate the interpolation problem we need first to define a class of positive real functions of low complexity.

3.1. The class $\mathcal{F}_{+}(n)$. An $\ell \times \ell$ matrix-valued rational function $F$ that is analytic in the closed unit disc $\overline{\mathbb{D}}:=\{z \in \mathbb{C}:|z| \leq 1\}$ is called strictly positive real if the spectral density function

$$
\Phi\left(e^{i \theta}\right):=\Re\left\{F\left(e^{i \theta}\right)\right\}
$$

is positive definite for all $\theta \in[-\pi, \pi]$. Here,

$$
\Re\{F(z)\}:=\frac{1}{2}\left[F(z)+F^{*}(z)\right], \text { where } F^{*}(z):=\overline{F\left(\bar{z}^{-1}\right)^{\top}},
$$

is the Hermitian generalization of the real part in the scalar case. Let $\mathcal{C}_{+}$be the class of all such functions. If $F$ belongs to $\mathcal{C}_{+}$, then so does $F^{-1}$. In particular, $F$ is outer, i.e., all its poles and zeros are located in $\mathbb{D}^{c}$, the complement of $\overline{\mathbb{D}}$.

Strictly positive real functions abound in control, circuit theory and signal processing, where they often represent transfer functions of filters or closed-loop control systems. Since design limitations require such devises to be of bounded complexity, the class $\mathcal{C}_{+}$needs to be restricted to accommodate appropriate complexity constraints. Typically, the McMillan degree needs to be bounded.

To this end, first note that, to each $F \in \mathcal{C}_{+}$, there corresponds an outer $\ell \times \ell$ matrix-valued function $V$ such that

$$
V(z) V^{*}(z)=\Phi(z):=\Re\{F(z)\},
$$

which is unique modulo an orthogonal transformation. Determining $V$ from $F$ is a spectral factorization problem, which can be solved by determining the stabilizing solution of an algebraic Riccati equation (see, e.g., [14]). Conversely, if

$$
V(z)=z C(I-z A)^{-1} B+D
$$

is any minimal realization of $V$, appealing to the equations of the Kalman-YakubovichPopov Lemma, there is a unique $F$ satisfying (3.2), and it is given by

$$
F(z)=2 z C(I-z A)^{-1}\left(A X C^{*}+B D^{*}\right)+C X C^{*}+D D^{*},
$$

where $X$ is the unique solution to the Lyapunov equation

$$
X=A X A^{*}+B B^{*} .
$$

Moreover, $V$ is a proper rational function of the same McMillan degree as $F$, and so is the inverse $V^{-1}$.

Let the polynomial $\rho$ be the least common denominator of all entries in $V^{-1}$. Then there is a matrix polynomial $R$ of the same degree as $\rho$ such that $V^{-1}=R / \rho$, and consequently

$$
V(z)=\rho(z) R(z)^{-1}
$$


In this representation, the degree $r:=\operatorname{deg} \rho$ is uniquely determined by $F$; to emphasize this we write $r(F)$. Now, define the class

$$
\mathcal{F}_{+}(n):=\left\{F \in \mathcal{C}_{+} \mid r(F) \leq n\right\} .
$$

All functions $F \in \mathcal{F}_{+}(n)$ have McMillan degree at most $\ell n$, but, although this is a nongeneric situation, there are $F \in \mathcal{C}_{+}$of McMillan degree at most $\ell n$ that do not belong to $\mathcal{F}_{+}(n)$. In fact, the standard observable (standard reachable) realization of $V^{-1}$ has dimension $\ell r$ (see, e.g., [3, p. 106]), and consequently $V^{-1}$, and hence $F$, has McMillan degree at most $\ell r$. Moreover, the standard observable realization may not be minimal, so there is a thin set of $F \in \mathcal{C}_{+}$of McMillan degree at most $\ell n$ for which $r(F)>n$.

3.2. Problem formulation. Suppose that we are given a set

$$
\mathcal{Z}:=\left\{z_{0}, z_{1}, \ldots, z_{n}\right\} \subset \mathbb{D}
$$

of $n+1$ interpolation points in the open unit disc $\mathbb{D}$. These points need not be distinct, but, if a certain number is repeated, it occurs in sequence. We say that $z_{k}$ has multiplicity $\nu$ if $z_{k}=z_{k+1}=\cdots=z_{k+\nu-1}$ and no other point takes this value. Moreover, suppose we have a set of $n+1$ matrix-valued interpolation values

$$
\mathcal{W}:=\left\{W_{0}, W_{1}, \ldots, W_{n}\right\} \subset \mathbb{C}^{\ell \times \ell} .
$$

We assume for convenience that $z_{0}=0$ and that $W_{0}$ is real and symmetric.

Now, consider the problem to find a function $F \in \mathcal{F}_{+}(n)$ that satisfies the interpolation condition

$$
F\left(z_{k}\right)=W_{k}
$$

for each $k$ such that $z_{k}$ has multiplicity one and

$$
\frac{1}{j !} F^{(j)}\left(z_{k}\right)=W_{k+j}, \quad j=0,1, \ldots, \nu-1,
$$

whenever $z_{k}$ has multiplicity $\nu$ and $z_{k}=z_{k+1}=\cdots=z_{k+\nu-1}$.

This is a matrix-valued Nevanlinna-Pick interpolation problem with a nonclassical complexity constraint, namely the condition that the interpolant $F$ must belong to the set $\mathcal{F}_{+}(n)$. In the scalar case $\ell=1$, this is a degree constraint, and the problem has been studied in $[6,7,11,12,26,27]$. In the present multivariable setting, this complexity constraint is not merely a degree constraint, as pointed out above. In fact, although all $F \in \mathcal{F}_{+}(n)$ have degree at most $\ell n, \mathcal{F}_{+}(n)$ does not contain all such functions.

This problem could be reformulated as a generalized moment problem. To see this, note that, by the matrix version of the Herglotz Theorem [18], any $F \in \mathcal{F}_{+}(n)$ could be represented as

$$
F(z)=\frac{1}{2 \pi} \int_{-\pi}^{\pi} \frac{e^{i \theta}+z}{e^{i \theta}-z} \Phi\left(e^{i \theta}\right) d \theta,
$$

where $\Phi$ is given by (3.1). Since therefore

$$
\frac{1}{j !} F^{(j)}(z)=\frac{1}{2 \pi} \int_{-\pi}^{\pi} \frac{2 e^{i \theta}}{\left(e^{i \theta}-z\right)^{j+1}} \Phi\left(e^{i \theta}\right) d \theta, \quad j=1,2, \ldots
$$


the interpolation conditions (3.10) and (3.11) can be combined to

$$
\frac{1}{2 \pi} \int_{-\pi}^{\pi} \alpha_{k}\left(e^{i \theta}\right) \Phi\left(e^{i \theta}\right) d \theta=W_{k}, \quad k=0,1, \ldots, n,
$$

where $\alpha_{k}$ is defined as

$$
\alpha_{k}(z)=\frac{z+z_{k}}{z-z_{k}}
$$

when $z_{k}$ has multiplicity one, and as

$$
\alpha_{k}(z)=\frac{z+z_{k}}{z-z_{k}}, \quad \alpha_{k+j}(z)=\frac{2 z}{\left(z-z_{k}\right)^{j+1}}, \quad j=1,2, \ldots, \nu-1,
$$

when $z_{k}$ has multiplicity $\nu$ and $z_{k}=z_{k+1}=\cdots=z_{k+\nu-1}$. In particular, since $z_{0}=0$, $\alpha_{0}=1$. Consequently, the Nevanlinna-Pick interpolation problem with complexity constraint formulated above is equivalent to finding an $F \in \mathcal{F}_{+}(n)$ satisfying (3.13).

3.3. A necessary and sufficient condition for existence of solutions. Clearly the problem posed above does not have a solution for all choices of $\mathcal{W}$. Next, we shall therefore determine what conditions need to be imposed on the interpolation values $W_{0}, W_{1}, \ldots, W_{n}$. To this end, we first introduce the class $\mathcal{Q}(\ell, n)$ of $\ell \times \ell$ matrix-valued generalized pseudo-polynomials

$$
Q(z)=\Re\left\{\sum_{k=0}^{n} Q_{k} \alpha_{k}(z)\right\}
$$

with coefficients $Q_{k} \in \mathbb{C}^{\ell \times \ell}$ and $Q_{0}$ real and symmetric, and then we define the subset

$$
Q_{+}(\ell, n):=\left\{Q \in \mathcal{Q}(\ell, n) \mid Q\left(e^{i \theta}\right)>0 \text { for all } \theta \in[-\pi, \pi]\right\}
$$

consisting of those $Q \in \mathcal{Q}(\ell, n)$ that are positive on the unit circle.

Definition 3.1. Given the interpolation points $\mathcal{Z}$, the sequence $\mathcal{W}$ of interpolation values is positive if

$$
\operatorname{Re}\left\{\sum_{k=0}^{n} \operatorname{tr}\left(Q_{k} W_{k}\right)\right\}>0,
$$

for all matrix sequences $Q_{0}, Q_{1}, \ldots, Q_{n}$ such that the pseudo-polynomial $Q$ defined by (3.16) belongs to $\mathcal{Q}_{+}(\ell, n)$. Let $\mathfrak{W}_{+}(\ell, n)$ be the class of all such positive sequences. Here $\operatorname{tr}\{A\}$ denotes the trace of the square matrix $A$.

Theorem 3.1. There exists an $F \in \mathcal{F}_{+}(n)$ satisfying the interpolation condition (3.13) if and only if $\mathcal{W}$ is positive.

The proof that positivity of $\mathcal{W}$ is necessary is classical. To see this, just note that (3.13) implies that

$$
\operatorname{Re}\left\{\sum_{k=0}^{n} \operatorname{tr}\left(Q_{k} W_{k}\right)\right\}=\frac{1}{2 \pi} \int_{-\pi}^{\pi} \operatorname{tr}\left\{Q\left(e^{i \theta}\right) \Phi\left(e^{i \theta}\right)\right\} d \theta,
$$

which is positive whenever $Q \in \mathcal{Q}_{+}(\ell, n)$. In Section 5 we shall prove that this condition is also sufficient. 
Now, it will be useful to represent (3.18) in terms of the inner product

$$
\langle A, B\rangle:=\frac{1}{2 \pi} \int_{-\pi}^{\pi} \operatorname{tr} A^{*}\left(e^{i \theta}\right) B\left(e^{i \theta}\right) d \theta
$$

between two $\ell \times \ell$ matrix-valued $\mathcal{L}^{2}$ functions $A$ and $B$.

Proposition 3.2. Let $W_{+}: \mathbb{D} \rightarrow \mathbb{C}^{\ell \times \ell}$ be an arbitrary analytic function satisfying the interpolation condition

$$
\frac{1}{2 \pi} \int_{-\pi}^{\pi} \alpha_{k}\left(e^{i \theta}\right) W\left(e^{i \theta}\right) d \theta=W_{k}, \quad k=0,1, \ldots, n
$$

with $W:=\Re\left\{W_{+}\right\}$. Then

$$
\operatorname{Re}\left\{\sum_{k=0}^{n} \operatorname{tr}\left(Q_{k} W_{k}\right)\right\}=\langle Q, W\rangle .
$$

Proof. Given any $W$ defined as in the proposition, we obtain

$$
\begin{aligned}
\langle Q, W\rangle & =\frac{1}{2 \pi} \int_{-\pi}^{\pi} \operatorname{tr}\left(Q\left(e^{i \theta}\right) W\left(e^{i \theta}\right)\right) d \theta \\
& =\operatorname{Re} \sum_{k=0}^{n} \operatorname{tr}\left(Q_{k} \frac{1}{2 \pi} \int_{-\pi}^{\pi} \alpha_{k}\left(e^{i \theta}\right) W\left(e^{i \theta}\right) d \theta\right) \\
& =\operatorname{Re}\left\{\sum_{k=0}^{n} \operatorname{tr}\left(Q_{k} W_{k}\right)\right\},
\end{aligned}
$$

which establishes (3.22).

Note that $W_{+}$need not be in $\mathcal{F}_{+}(n)$ and that such a function is easy to compute. However, in this paper $W$ is merely a notational device and will never have to be determined.

3.4. The generalized Pick condition. The positivity condition in Theorem 3.1 is a generalized Pick condition. To see this, let $\Gamma(z)$ be any minimum-phase solution of the spectral factorization problem

$$
\Gamma(z) \Gamma^{*}(z)=Q(z) .
$$

Then, introducing the vector of Cauchy type kernels

$$
G(z):=\left[\begin{array}{llll}
g_{0}^{*}(z) & g_{1}^{*}(z) & \cdots & g_{n}^{*}(z)
\end{array}\right],
$$

where

$$
g_{k}(z)=\frac{1}{2}\left(\alpha_{k}(z)+1\right)
$$

for those $k$ for which $\alpha_{k}$ is given by (3.14) and

$$
g_{k}(z)=\frac{1}{2} \alpha_{k}(z)
$$

for all other $k, \Gamma(z)$ has a representation

$$
\Gamma(z)=\left(G(z) \otimes I_{\ell}\right) \Gamma
$$


for some matrix $\Gamma \in \mathbb{C}^{\ell(n+1) \times \ell}$. Now, let $W$ be defined as in Proposition 3.2. Then (3.23) yields

$$
\langle Q, W\rangle=\langle\Gamma, W \Gamma\rangle=\operatorname{tr}\left\{\boldsymbol{\Gamma}^{*} \boldsymbol{\Pi} \Gamma\right\}
$$

where $\boldsymbol{\Pi}$ is the generalized Pick matrix

$$
\Pi:=\frac{1}{2 \pi} \int_{-\pi}^{\pi}\left(G^{*}\left(e^{i \theta}\right) \otimes I_{\ell}\right) W\left(e^{i \theta}\right)\left(G\left(e^{i \theta}\right) \otimes I_{\ell}\right) d \theta
$$

where $A \otimes B$ is the Kronecker product of $A$ and $B$.

Hence we have the following corollary of Theorem 3.1.

Corollary 3.3. The sequence $\mathcal{W}$ is positive if and only if the Pick matrix (3.27) is positive definite.

When $z_{k}$ has multiplicity $\nu$ and $z_{k}=z_{k+1}=\cdots=z_{k+\nu-1}$, we have

$$
\frac{1}{j !} F^{(j)}\left(z_{k}\right)=\frac{1}{2 \pi} \int_{-\pi}^{\pi} g_{k+j}\left(e^{i \theta}\right) F\left(e^{i \theta}\right) d \theta, \quad j=0,1, \ldots, \nu-1,
$$

for any function $F$ that is analytic in the unit disc $\mathbb{D}$. Using this Cauchy integral formula, a straight-forward, but tedious, calculation yields

$$
\boldsymbol{\Pi}=\frac{1}{2}\left[\boldsymbol{W}\left(\boldsymbol{S} \otimes I_{\ell}\right)+\left(\boldsymbol{S} \otimes I_{\ell}\right) \boldsymbol{W}^{*}\right],
$$

where $\boldsymbol{S}$ is the Gramian

$$
\boldsymbol{S}:=\frac{1}{2 \pi} \int_{-\pi}^{\pi} G^{*}\left(e^{i \theta}\right) G\left(e^{i \theta}\right) d \theta
$$

and $\boldsymbol{W}$ is a block diagonal matrix consisting of one block

$$
\left[\begin{array}{ccc}
W_{k} & & \\
\vdots & \ddots & \\
W_{k+\nu-1} & \cdots & W_{k}
\end{array}\right]
$$

for each distinct point in $z$ taken in order. The Gramian (3.29) can be determined by solving the Lyapunov equation

$$
\boldsymbol{S}-\boldsymbol{A} \boldsymbol{S} \boldsymbol{A}^{*}=\boldsymbol{b} \boldsymbol{b}^{\top}
$$

where $\boldsymbol{A}$ is a block diagonal matrix formed from the $\nu \times \nu$ blocks

$$
A_{k}:=\left[\begin{array}{cccc}
z_{k} & & & \\
1 & z_{k} & & \\
& \ddots & \ddots & \\
& & 1 & z_{k}
\end{array}\right],
$$

and $\boldsymbol{b}$ is a column vector of ones and zeros in which the ones occur for those $k$ for which (3.14) holds.

Specializing to the case when all interpolation points have multiplicity one, we obtain the classical Pick matrix

$$
\boldsymbol{\Pi}=\frac{1}{2}\left[\frac{W_{i}+W_{j}^{*}}{1-z_{i} \bar{z}_{j}}\right]_{i, j=0}^{n} .
$$


On the other hand, when there is only one interpolation point with multiplicity $n+1$ located at the origin, as in the classical Carathéodory extension problem, the Pick matrix is the block Toeplitz matrix

$$
\boldsymbol{\Pi}=\frac{1}{2}\left[\begin{array}{cccc}
W_{0}+W_{0}^{*} & W_{1}^{*} & \cdots & W_{n}^{*} \\
W_{1} & W_{0}+W_{0}^{*} & \cdots & W_{n-1}^{*} \\
\vdots & \vdots & \ddots & \vdots \\
W_{n} & W_{n-1} & \cdots & W_{0}+W_{0}^{*}
\end{array}\right] .
$$

See, e.g., $[19,20]$.

\section{MAIN THEOREMS}

To motivate the approach taken in this paper, we first consider the special case when $z_{0}=z_{1}=\cdots=z_{n}=0$, i.e.,

$$
\alpha_{0}(z)=1, \quad \alpha_{k}(z)=2 z^{-k}, k=1, \ldots, n,
$$

which is of particular interest in signal processing and identification. In this case the generalized Pick condition reduces to a Toeplitz condition, as described above. In particular, the interpolant that maximizes the entropy gain

$$
\frac{1}{2 \pi} \int_{-\pi}^{\pi} \log \operatorname{det} \Phi\left(e^{i \theta}\right) d \theta
$$

is the maximum entropy solution discussed in Section 2. Like $\mathcal{W}$, the cepstral coefficients [43],

$$
c_{k}:=\frac{1}{2 \pi} \int_{-\pi}^{\pi} \alpha_{k}\left(e^{i \theta}\right) \log \operatorname{det} \Phi\left(e^{i \theta}\right) d \theta, \quad k=0,1, \ldots, n,
$$

can be observed. In the scalar case $\ell=1$, it was noted in $[4,5]$ that the entropy gain (4.1) is precisely the zeroth cepstral coefficient $c_{0}$ and that the cepstral coefficients (4.2) together with the covariance data $\mathcal{W}$ form local coordinates of $\mathcal{F}_{+}(n)$. This observation led to maximizing linear combinations of the cepstral coefficients instead.

In this paper we shall apply the same strategy to the multivariable Nevanlinna-Pick problem when $\alpha_{0}, \alpha_{1}, \ldots, \alpha_{n}$ are given by (3.14) and (3.15). Accordingly, we consider the problem of maximizing some linear combination

$$
\operatorname{Re}\left\{\sum_{k=0}^{n} p_{k} c_{k}\right\}
$$

of the coefficients (4.2), which, in this more general setting, will be referred to as the generalized cepstral coefficients. Introducing the generalized pseudo-polynomial

$$
P(z):=\operatorname{Re}\left\{\sum_{k=0}^{n} p_{k} \alpha_{k}(z)\right\},
$$

(4.3) can be written as the generalized entropy gain

$$
\mathbb{I}_{P}(\Phi):=\frac{1}{2 \pi} \int_{-\pi}^{\pi} P\left(e^{i \theta}\right) \log \operatorname{det} \Phi\left(e^{i \theta}\right) d \theta,
$$

which we want to maximize over the class $\mathcal{S}_{+}^{\ell \times \ell}$ of (not necessarily rational) bounded, coercive spectral densities $\Phi$, i.e., bounded $\Phi$ such that $\Phi^{-1}$ is also bounded. Just as 
in $[4,5]$ we must require $P(z)$ to be positive on the unit circle, i.e., $P \in Q_{+}(1, n)$, in order for a maximum of $\mathbb{I}_{P}(\Phi)$ to exist. In fact, the following theorem establishes a complete parameterization of all interpolants $F \in \mathcal{F}_{+}(n)$ in terms of the generalized pseudo-polynomial $P \in \mathcal{Q}_{+}(1, n)$.

Theorem 4.1. Let $P \in Q_{+}(1, n)$, and suppose that the positivity condition (3.18) holds. Then the maximization problem

$$
\max _{\Phi \in \mathcal{S}_{+}^{\ell \times \ell}} \mathbb{I}_{P}(\Phi) \quad \text { subject to } \frac{1}{2 \pi} \int_{-\pi}^{\pi} \alpha_{k}\left(e^{i \theta}\right) \Phi\left(e^{i \theta}\right) d \theta=W_{k}, k=0,1, \ldots, n,
$$

has a unique optimal solution, and it takes the form

$$
\Phi(z)=P(z) Q(z)^{-1},
$$

where $Q \in \mathcal{Q}_{+}(\ell, n)$. Via (3.12) this establishes a one-one correspondence between interpolants $F \in \mathcal{F}_{+}(n)$ and $P \in \mathcal{Q}_{+}(1, n)$.

This is a constrained optimization problem over the infinite-dimensional space $\mathcal{S}_{+}^{\ell \times \ell}$, but with finitely many constraints. In analogy with [7] we shall demonstrate in Section 5 that there is a dual optimization problem over a finite-dimensional space, namely the problem to find a $Q \in Q_{+}(\ell, n)$ that minimizes the functional

$$
\mathbb{J}_{P}(Q)=\langle Q, W\rangle-\frac{1}{2 \pi} \int_{-\pi}^{\pi} P\left(e^{i \theta}\right) \log \operatorname{det} Q\left(e^{i \theta}\right) d \theta .
$$

From Definition 3.1 and Theorem 3.1 we recall that the sequence $\mathcal{W}$ of interpolation values is positive if and only if

$$
\langle Q, W\rangle>0 \text { for all } Q \in \mathcal{Q}_{+}(\ell, n) .
$$

Theorem 4.2. Let $P \in Q_{+}(1, n)$, and suppose that the positivity condition (4.9) holds. Then the minimization problem

$$
\min _{Q \in \mathcal{Q}_{+}(\ell, n)} \mathbb{J}_{P}(Q)
$$

has a unique optimal solution. For the optimal solution $\hat{Q}$, the interpolant $F \in \mathcal{F}_{+}(n)$ corresponding to $P$ is given by

$$
F(z)=\frac{1}{2 \pi} \int_{-\pi}^{\pi} \frac{e^{i \theta}+z}{e^{i \theta}-z} P\left(e^{i \theta}\right) \hat{Q}\left(e^{i \theta}\right)^{-1} d \theta
$$

The optimal solution $\hat{Q}$ depends smoothly on the interpolation data $\mathcal{W}$ and on $P$. In particular, the map $\mathcal{J}: \mathcal{Q}_{+}(\ell, n) \rightarrow \mathfrak{W}_{+}(\ell, n)$ with components

$$
\mathcal{J}_{k}(Q):=\frac{1}{2 \pi} \int_{-\pi}^{\pi} \alpha_{k}\left(e^{i \theta}\right) P\left(e^{i \theta}\right) Q\left(e^{i \theta}\right)^{-1} d \theta, \quad k=0,1, \ldots, n,
$$

is a diffeomorphism.

It is easy to see that any $P \in Q_{+}(1, n)$ has a unique representation of the form

$$
P(z)=\frac{\rho(z) \rho^{*}(z)}{\tau(z) \tau^{*}(z)}
$$


where

$$
\tau(z):=\prod_{k=1}^{n}\left(1-\bar{z}_{k} z\right)
$$

belongs to the class $\mathfrak{S}_{+}$of polynominals with all roots in $\mathbb{D}^{c}$, and where

$$
\rho(z)=\rho_{0}+\rho_{1} z+\cdots+\rho_{n} z^{n}
$$

is an arbitrary polynomial in $\mathfrak{S}_{+}$. Hence, there is a one-to-one correspondence between interpolant $F \in \mathcal{F}_{+}(n)$ and $\rho \in \mathfrak{S}_{+}$, and this parameterization is smooth, in fact a diffeomorphism. The parameters $\rho_{0}, \rho_{1}, \ldots, \rho_{n}$ can therefore serve as "tuning parameters" in robust control and other applications. The interpolant $F$ can be determined from the solution to the dual optimization problem (4.10) in a fashion to be described in Section 6.

Similarly, any $Q \in \mathcal{Q}_{+}(\ell, n)$ has a representation (3.23), i.e. $Q(z)=\Gamma(z) \Gamma^{*}(z)$, unique up to an orthogonal transformation, where

$$
\Gamma(z)=\tau(z)^{-1} R(z)
$$

and the $\ell \times \ell$ matrix polynomial

$$
R(z)=R_{0}+R_{1} z+\cdots+R_{n} z^{n}
$$

are outer. In Section 6 we assume that the interpolation data $z, \mathcal{W}$ is self-conjugate, and the matrix coefficients are real. We also show that the dual optimization problem can be reformulated in terms of $R(z)$ so that, in particular, the spectral factorization step and complex number calculations are avoided.

Consequently, for each choice of tuning parameters $\rho_{0}, \rho_{1}, \ldots, \rho_{n}$, the dual optimization problem provides an essentially unique matrix polynomial (4.17) so that

$$
V(z):=\rho(z) R(z)^{-1}
$$

is an outer spectral factor of $\Phi=P Q^{-1}$. Forming a minimal realization (3.3) of (4.18), the corresponding interpolant $F \in \mathcal{F}_{+}(n)$ is given by (3.4).

\section{Proofs in the CONTEXT OF DUAlity theory}

To solve the problem (4.6), we form the Lagrangian

$$
L(\Phi, Q):=\mathbb{I}_{P}(\Phi)+\operatorname{Re}\left\{\sum_{k=0}^{n} \sum_{i=1}^{\ell} \sum_{j=1}^{\ell} q_{k}^{j i}\left[w_{k}^{i j}-\frac{1}{2 \pi} \int_{-\pi}^{\pi} \alpha_{k}\left(e^{i \theta}\right) \Phi_{i j}\left(e^{i \theta}\right) d \theta\right]\right\},
$$

where $w_{k}^{i j}$ and $\Phi_{i j}$ are the matrix components of $W_{k}$ and $\Phi$ respectively, and then solve the dual problem to minimize

$$
\sup _{\Phi \in \mathcal{S}_{+}^{\ell \times \ell}} L(\Phi, Q)
$$

with respect to the Lagrange multipliers $q_{k}^{i j}$, which are complex numbers except when $k=0$ when they are real and $q_{0}^{j i}=q_{0}^{i j}$. For each $k=0,1, \ldots, n$, let $Q_{k}$ be the $\ell \times \ell$ matrix $\left[q_{k}^{i j}\right]_{i, j=1}^{\ell}$, and form the generalized pseudo-polynomial (3.16). Then, using the identity (3.22), the Lagrangian can be written by

$$
L(\Phi, Q)=\frac{1}{2 \pi} \int_{-\pi}^{\pi} P\left(e^{i \theta}\right) \log \operatorname{det} \Phi\left(e^{i \theta}\right) d \theta+\langle Q, W\rangle-\frac{1}{2 \pi} \int_{-\pi}^{\pi} \operatorname{tr}\left\{Q\left(e^{i \theta}\right) \Phi\left(e^{i \theta}\right)\right\} d \theta
$$


Clearly, the Lagrangian will be unbounded if $Q$ is allowed to take negative values on the unit circle. Hence, we determine the supremum for each $Q \in \mathcal{Q}_{+}(\ell, N)$. To this end, we want to determine a $\Phi$ such that the directional derivative

$$
\begin{aligned}
\delta L(\Phi, Q ; \delta \Phi) & :=\lim _{\epsilon \rightarrow 0} \frac{L(\Phi+\epsilon \delta \Phi, Q)-L(\Phi, Q)}{\epsilon} \\
& =\frac{1}{2 \pi} \int_{-\pi}^{\pi} P\left(e^{i \theta}\right) \lim _{\epsilon \rightarrow 0} \frac{1}{\epsilon} \log \left[\frac{\operatorname{det}(\Phi+\epsilon \delta \Phi)}{\operatorname{det} \Phi}\right] d \theta-\frac{1}{2 \pi} \int_{-\pi}^{\pi} \operatorname{tr}\{Q \delta \Phi\} d \theta
\end{aligned}
$$

equals zero in all directions $\delta \Phi$ such that $\Phi+\epsilon \delta \Phi \in \mathcal{S}_{+}^{\ell \times \ell}$ for some $\epsilon>0$. However,

$$
\log \left[\frac{\operatorname{det}(\Phi+\epsilon \delta \Phi)}{\operatorname{det} \Phi}\right]=\log \operatorname{det}\left(I+\epsilon \Phi^{-1} \delta \Phi\right)=\log \prod_{j=1}^{\ell} \mu_{j}=\sum_{j=1}^{\ell} \log \mu_{j},
$$

where $\mu_{j}\left(e^{i \theta}\right), j=1,2, \ldots, \ell$, are the eigenvalues of $\left[I+\epsilon \Phi\left(e^{i \theta}\right)^{-1} \delta \Phi\left(e^{i \theta}\right)\right]$. Since $\mu_{j}=1+\epsilon \lambda_{j}, j=1,2, \ldots, \ell$, where $\lambda_{1}\left(e^{i \theta}\right), \lambda_{2}\left(e^{i \theta}\right), \ldots, \lambda_{\ell}\left(e^{i \theta}\right)$ are the eigenvalues of $\Phi\left(e^{i \theta}\right)^{-1} \delta \Phi\left(e^{i \theta}\right)$, and $\log \left(1+\epsilon \lambda_{j}\right)=\epsilon \lambda_{j}+O\left(\epsilon^{2}\right)$, we have

$$
\lim _{\epsilon \rightarrow 0} \frac{1}{\epsilon} \log \left[\frac{\operatorname{det}(\Phi+\epsilon \delta \Phi)}{\operatorname{det} \Phi}\right]=\sum_{j=1}^{\ell} \lambda_{j}=\operatorname{tr}\left(\Phi^{-1} \delta \Phi\right) .
$$

Consequently, in terms of the inner product the directional derivative can be written as

$$
\delta L(\Phi, Q ; \delta \Phi)=\left\langle\delta \Phi, P \Phi^{-1}-Q\right\rangle
$$

which equals zero for all $\delta \Phi$ if and only if

$$
\Phi=P Q^{-1} .
$$

Inserting this into (5.1) we obtain

$$
\mathbb{J}_{P}(Q)+\frac{\ell}{2 \pi} \int_{-\pi}^{\pi} P\left(e^{i \theta}\right)\left(\log P\left(e^{i \theta}\right)-1\right) d \theta,
$$

where

$$
\mathbb{J}_{P}(Q)=\langle Q, W\rangle-\frac{1}{2 \pi} \int_{-\pi}^{\pi} P\left(e^{i \theta}\right) \log \operatorname{det} Q\left(e^{i \theta}\right) d \theta .
$$

Hence, modulo an additive constant, $\mathbb{J}_{P}$ is precisely the dual function.

We want to show that this functional is strictly convex and that it has a unique minimum in $\mathcal{Q}_{+}(\ell, n)$. To this end, we form the directional derivative

$$
\begin{aligned}
\delta \mathbb{J}_{P}(Q ; \delta Q) & :=\lim _{\epsilon \rightarrow 0} \frac{\mathbb{J}_{P}(Q+\epsilon \delta Q)-\mathbb{J}_{P}(Q)}{\epsilon} \\
& =\langle\delta Q, W\rangle-\frac{1}{2 \pi} \int_{-\pi}^{\pi} P\left(e^{i \theta}\right) \lim _{\epsilon \rightarrow 0} \frac{1}{\epsilon} \log \left[\frac{\operatorname{det}(Q+\epsilon \delta Q)}{\operatorname{det} Q}\right] d \theta \\
& =\left\langle\delta Q, W-P Q^{-1}\right\rangle
\end{aligned}
$$

where we have performed the same calculation as in (5.2). We need to determine a $Q \in Q_{+}(\ell, n)$ such that

$$
\delta \mathbb{J}_{P}(Q ; \delta Q)=0
$$


for all $\delta Q$ of the form

$$
\delta Q\left(e^{i \theta}\right)=\Re\left\{\sum_{k=0}^{n} \delta Q_{k} \alpha_{k}\left(e^{i \theta}\right)\right\},
$$

where $\delta Q_{k}, k=0,1, \ldots, n$, are arbitrary complex $\ell \times \ell$ matrices, except for $\delta Q_{0}$ that is real and symmetric. Inserting (5.8) into (5.6), we obtain

$$
\begin{aligned}
\delta \mathbb{J}_{P}(Q ; \delta Q) & =\operatorname{Re}\left\{\sum_{k=0}^{n} \operatorname{tr}\left(\delta Q_{k} \frac{1}{2 \pi} \int_{-\pi}^{\pi} \alpha_{k}\left(e^{i \theta}\right)\left[W\left(e^{i \theta}\right)-P\left(e^{i \theta}\right) Q\left(e^{i \theta}\right)^{-1}\right] d \theta\right)\right\} \\
& =\operatorname{Re}\left\{\sum_{k=0}^{n} \operatorname{tr}\left(\delta Q_{k}\left[W_{k}-\mathcal{J}_{k}(Q)\right]\right)\right\}
\end{aligned}
$$

where $\mathcal{J}_{0}(Q), \mathcal{J}_{1}(Q), \ldots, \mathcal{J}_{n}(Q)$ are defined as in (4.12). Hence (5.7) cannot hold for all $\delta Q$ unless $\mathcal{J}_{k}(Q)=W_{k}, k=0,1, \ldots, n$, that is, unless (5.4) satisfies the interpolation condition (3.13). To see this, for an arbitrary $(k, i, j)$ with $k \neq 0$, take all components of $\delta Q_{0}, \delta Q_{1}, \ldots, \delta Q_{n}$ equal to zero except $\delta q_{k}^{i j}$, which we take to be $\lambda+i \mu$ with $\lambda$ and $\mu$ arbitrary. Then, letting $u_{k}^{i j}$ be the real part and $v_{k}^{i j}$ the imaginary part of $w_{k}^{i j}-\mathrm{J}_{k}^{i j}(Q)$, we obtain

$$
\delta \mathbb{J}_{P}(Q ; \delta Q)=\operatorname{Re}\left\{(\lambda+i \mu)\left(u_{k}^{i j}+i v_{k}^{i j}\right)\right\}=\lambda u_{k}^{i j}-\mu v_{k}^{i j},
$$

and hence $w_{k}^{i j}=\mathfrak{J}_{k}^{i j}(Q)$, as claimed. If $k=0, \mu$ and $v_{k}^{i j}$ equal to zero, so the same conclusion follows. It remains to show that there is a $Q \in Q_{+}(\ell, n)$ such that (5.7) holds.

Theorem 5.1. Let $P \in \mathcal{Q}_{+}(1, n)$, and suppose that the positivity condition (3.18) holds. The dual functional $\mathbb{J}_{P}: \mathcal{Q}_{+}(\ell, n) \rightarrow \mathbb{R}$ is strictly convex and has a unique minimum $\hat{Q}$. Moreover,

$$
\frac{1}{2 \pi} \int_{-\pi}^{\pi} \alpha_{k}\left(e^{i \theta}\right) P\left(e^{i \theta}\right) \hat{Q}\left(e^{i \theta}\right)^{-1} d \theta=W_{k}, \quad k=0,1, \ldots, n
$$

Proof. To prove that $\mathbb{J}_{P}$ is strictly convex we form

$$
\begin{aligned}
\delta^{2} \mathbb{J}_{P}(Q ; \delta Q) & :=\lim _{\epsilon \rightarrow 0} \frac{\delta \mathbb{J}_{P}(Q+\epsilon \delta Q ; \delta Q)-\delta \mathbb{J}_{P}(Q ; \delta Q)}{\epsilon} \\
& =-\lim _{\epsilon \rightarrow 0} \frac{1}{\epsilon}\left\langle\delta Q, P\left[(Q+\epsilon \delta Q)^{-1}-Q^{-1}\right]\right\rangle \\
& =\lim _{\epsilon \rightarrow 0} \frac{1}{\epsilon}\left\langle\delta Q, P\left[I-\left(I+\epsilon Q^{-1} \delta Q\right)^{-1}\right] Q^{-1}\right\rangle
\end{aligned}
$$

However,

$$
\left(I+\epsilon Q^{-1} \delta Q\right)^{-1}=I-\epsilon Q^{-1} \delta Q+O\left(\epsilon^{2}\right)
$$

for sufficiently small $\epsilon>0$, and hence

$$
\delta^{2} \mathbb{J}_{P}(Q ; \delta Q)=\left\langle\delta Q, P Q^{-1} \delta Q Q^{-1}\right\rangle
$$

Now, since $Q \in Q_{+}(\ell, n)$ is positive definite on the unit circle, there is a nonsingular matrix function $S$ such that $Q^{-1}=S S^{*}$. Then, using the commuting property of the trace, we have

$$
\operatorname{tr}\left(\delta Q Q^{-1} \delta Q Q^{-1}\right)=\operatorname{tr}\left(S^{*} \delta Q S S^{*} \delta Q S\right)
$$


and hence

$$
\delta^{2} \mathbb{J}_{P}(Q ; \delta Q)=\left\langle S^{*} \delta Q S, P\left(S^{*} \delta Q S\right)\right\rangle \geq 0,
$$

taking the value zero if and only if $S^{*} \delta Q S=0$ or, equivalently, $\delta Q=0$. Consequently, the Hessian of $\mathbb{J}_{P}(Q)$ is positive definite for all $Q \in Q_{+}(\ell, n)$, implying that $\mathbb{J}_{P}$ is strictly convex, as claimed.

The rest of the proof is the same mutatis mutandis as the one in [8]. (Also see $[7,9,11]$.) Since the linear term $\langle Q, W\rangle$ is positive and linear growth is faster than logarithmic, the function $\mathbb{J}_{P}$ is proper, i.e., the inverse images of compact sets are compact. In particular, if we extend the function $\mathbb{J}_{P}$ to the boundary of $Q_{+}(\ell, n)$, it has compact sublevel sets. Consequently, $\mathbb{J}_{P}$ has a minimum, $\hat{Q}$, which is unique by strict convexity. We need to rule out that $\hat{Q}$ lies on the boundary. To this end, note that the boundary of $Q_{+}(\ell, n)$ consists of the $Q$ for which $\operatorname{det} Q$ has a zero on the unit circle, and for which the directional derivative $\delta \mathbb{J}_{P}(Q ; \delta Q)=-\infty$ for all $\delta Q$ pointing into $Q_{+}(\ell, n)$. See $[9$, Section 4] for details.

Therefore, since $Q_{+}(\ell, n)$ is an open set, $\delta \mathbb{J}_{P}(\hat{Q} ; \delta Q)=0$ for all $\delta Q$ of the form (5.8), and therefore (5.9) follows.

Theorem 5.2. Let $P \in Q_{+}(1, n)$, and suppose that the positivity condition (3.18) holds. The primal functional $\mathbb{I}_{P}: \mathcal{S}_{+}^{\ell \times \ell} \rightarrow \mathbb{R}$ is strictly concave, and there is a unique optimal solution $\hat{\Phi} \in \mathcal{S}_{+}^{\ell \times \ell} \rightarrow \mathbb{R}$ to the problem (4.6). The maximum $\hat{\Phi}$ takes the form

$$
\hat{\Phi}=P \hat{Q}^{-1}
$$

where $\hat{Q} \in \mathcal{Q}_{+}(\ell, n)$ is the optimal solution of the dual problem.

Proof. To show that $\mathbb{I}_{P}$ is strictly concave, we proceed as above. The calculation leading to (5.3) yields

$$
\delta \mathbb{I}_{P}(\Phi ; \delta \Phi)=\left\langle\delta \Phi, P \Phi^{-1}\right\rangle,
$$

and, following the lines of the corresponding proof in Theorem 5.1,

$$
\delta^{2} \mathbb{I}_{P}(\Phi ; \delta \Phi) \leq 0
$$

with equality if and only if $\delta \Phi=0$. Hence $\mathbb{I}_{P}$ is strictly concave, as claimed.

Let $\hat{Q}$ be the optimal solution of the dual problem. Then, since $\mathbb{I}_{P}$ is strictly concave, then so is $\Phi \mapsto L(\Phi, \hat{Q})$. Clearly $\hat{\Phi}:=P \hat{Q}^{-1}$ belongs to $\mathcal{S}_{+}^{\ell \times \ell}$, and, by (5.3), it is a stationary point of the map $\Phi \mapsto L(\Phi, \hat{Q})$. Hence

$$
L(\hat{\Phi}, \hat{Q}) \geq L(\Phi, \hat{Q}), \quad \text { for all } \Phi \in \mathcal{S}_{+}^{\ell \times \ell} .
$$

However, by Theorem 5.1, $\hat{\Phi}$ satisfies the interpolation condition (3.13), and consequently

$$
L(\hat{\Phi}, \hat{Q})=\mathbb{I}_{P}(\hat{\Phi}) .
$$

Therefore, it follows from (5.10) that

$$
\mathbb{I}_{P}(\Phi) \leq \mathbb{I}_{P}(\hat{\Phi})
$$

for all $\Phi \in \mathcal{S}_{+}^{\ell \times \ell}$ that satisfies the interpolation condition (3.13), establishing optimality of $\hat{\Phi}$.

Consequently, we have proved Theorem 4.1. To finish the proof of Theorem 4.2 it remains to establish that the map $\mathcal{J}: \mathcal{Q}_{+}(\ell, n) \rightarrow \mathfrak{W}_{+}(\ell, n)$ is a diffeomorphism. To this end, first note that $\mathcal{Q}_{+}(\ell, n)$ and $\mathfrak{W}_{+}(\ell, n)$ are both convex, open sets in 
$\mathbb{R}^{2 n \ell^{2}+\frac{1}{2} \ell(\ell+1)}$ and hence diffeomorphic to $\mathbb{R}^{2 n \ell^{2}+\frac{1}{2} \ell(\ell+1)}$. Moreover, the Jacobian of $\mathcal{J}$ is the Hessian of $\mathbb{J}_{P}$, which is positive definite on $\mathcal{Q}_{+}(\ell, n)$, as shown in the proof of Theorem 5.1. Hence, by Hadamard's global inverse function theorem [30], $\mathcal{J}$ is a diffeomorphism.

Finally, Theorem 3.1 is an immediate consequence of Theorem 4.1.

\section{Solving the DuAl optimization Problem}

Recall that, by Theorem 4.2, for each choice of $P \in Q_{+}(1, n)$, there is a unique solution to the basic interpolation problem of this paper, and this solution is obtained by determining the unique minimizer over $Q_{+}(\ell, n)$ of the dual functional

$$
\mathbb{J}_{P}(Q):=\langle Q, W\rangle-\langle\log \operatorname{det} Q, P\rangle .
$$

This functional has the property that its gradient is infinite on the boundary of $Q_{+}(\ell, n)$. This is precisely the property that buys us properness of the functional (4.12), and therefore it is essential in the proof of Theorem 4.2. However, from a computational point of view, this property is undesirable, especially if the minimum is close to the boundary. In fact, it adversely affects the accuracy of any Newton-type algorithm. For this reason, following [22, 40], we first reformulate the optimization problem to eliminate this property. This is done at the expense of global convexity, but the new functional is still locally strictly convex in a neighborhood of a unique minimizing point. Thus, if we were able to choose the initial point in the convexity region, a Newton method would work well. However, finding such an initial point is a highly nontrivial matter. Therefore, again following [22, 40], we want to design a homotopy continuation method that determines a sequence of points converging to the minimizing point.

6.1. Reformulating the optimization problem. In Section 2 we replaced the first term in (6.1) with a quadratic form by first defining the spectral factor $\Gamma(z)$ satisfying (3.23). Consequently, for each $Q=\Gamma \Gamma^{*}$, the right hand side of (6.1) can also be written as

$$
\operatorname{tr} \Gamma^{*} \Pi \Gamma-\left\langle\log \operatorname{det} \Gamma \Gamma^{*}, P\right\rangle,
$$

where $\boldsymbol{\Pi}$ is the generalized Pick matrix defined by (3.27) or, alternatively, by (3.28). Let us now assume that the interpolation data $(\mathcal{Z}, \mathcal{W})$ is self-conjugate so that space $Q_{+}(\ell, n)$ has dimension $\ell^{2} n+\frac{1}{2} \ell(\ell+1)$ and the matrix coefficients $R_{0}, R_{1}, \ldots, R_{n}$ in

$$
R(z):=\tau(z) \Gamma(z)=R_{0}+R_{1} z+\cdots+R_{n} z^{n}
$$

are real. We also assume that $R_{0}$ is upper triangular. Then, the space $\mathcal{R}_{+}(\ell, n)$ of all

$$
\boldsymbol{R}:=\left[\begin{array}{c}
R_{0} \\
\vdots \\
R_{n}
\end{array}\right] \in \mathbb{R}^{\ell(n+1) \times \ell}
$$

such that (6.2) is outer and $R\left(e^{i \theta}\right) R\left(e^{i \theta}\right)^{*}>0$ for all $\theta \in[-\pi, \pi]$ also has dimension $\ell^{2} n+\frac{1}{2} \ell(\ell+1)$. In view of $(3.25)$,

$$
R(z)=\tau(z) G(z) \boldsymbol{\Gamma}
$$

which defines a nonsingular linear transformation $T$ such that

$$
\Gamma=T \boldsymbol{R} .
$$


Under this change of coordinates, the Pick matrix becomes

$$
\boldsymbol{K}=T^{*} \boldsymbol{\Pi} T,
$$

and, since $\arg \operatorname{det} R\left(e^{-i \theta}\right)=-\arg \operatorname{det} R\left(e^{i \theta}\right)$, (6.1) can be written

$$
\mathbb{J}_{P}(Q)=\boldsymbol{J}_{P}(\boldsymbol{R})-2\langle\log \tau, P\rangle,
$$

where the new cost functional

$$
\boldsymbol{J}_{P}(\boldsymbol{R})=\operatorname{tr} \boldsymbol{R}^{\top} \boldsymbol{K} \boldsymbol{R}-2\langle\log \operatorname{det} R, P\rangle
$$

is defined on the space $\mathcal{R}_{+}(\ell, n)$.

Proposition 6.1. The functional $\boldsymbol{J}_{P}: \mathcal{R}_{+}(\ell, n) \rightarrow \mathbb{R}$ has a unique stationary point and is locally strictly convex about this point.

Proof. Since $\Gamma(z):=R(z) / \tau(z)$ is a uniquely defined (outer) spectral factor of $Q(z)$, the map $\Psi: \mathcal{R}_{+}(\ell, n) \rightarrow \mathcal{Q}_{+}(\ell, n)$ sending $\boldsymbol{R}$ to $Q(z)=\Theta(z) \boldsymbol{R} \boldsymbol{R}^{*} \Theta^{*}(z)$, where

$$
\Theta(z):=\frac{1}{\tau(z)}\left[\begin{array}{llll}
I_{\ell} & z I_{\ell} & \cdots & z^{n} I_{\ell}
\end{array}\right]
$$

is a bijection with first and second directional derivatives

$$
\begin{aligned}
& \delta \Psi(\boldsymbol{R} ; \delta \boldsymbol{R})=\Theta(z)\left(\boldsymbol{R}(\delta \boldsymbol{R})^{*}+(\delta \boldsymbol{R}) \boldsymbol{R}^{*}\right) \Theta^{*}(z) \\
& \delta^{2} \Psi(\boldsymbol{R} ; \delta \boldsymbol{R})=2 \Theta(z)\left((\delta \boldsymbol{R})(\delta \boldsymbol{R})^{*}\right) \Theta^{*}(z) .
\end{aligned}
$$

Now, $\delta \boldsymbol{R} \mapsto \delta \Psi(\boldsymbol{R} ; \delta \boldsymbol{R})$ is an injective linear map between Euclidean spaces of the same dimension, and hence it is bijective. In fact, since det $R(z)$ has all its roots in the complement of the closed unit disc, the homogeneous equation

$$
R(z) \Delta^{*}(z)+\Delta(z) R^{*}(z) \equiv 0, \quad \Delta(z):=\Theta(z) \delta \boldsymbol{R},
$$

has a unique solution $\Delta(z) \equiv 0$. (See Lemma A.1 in Appendix A.) Therefore, since

$$
\boldsymbol{J}_{P}(\boldsymbol{R})=\mathbb{J}_{P}(\Psi(\boldsymbol{R}))+2\langle\log \tau, P\rangle,
$$

the directional derivative

$$
\delta \boldsymbol{J}_{P}(\boldsymbol{R} ; \delta \boldsymbol{R})=\delta \mathbb{J}_{P}(\Psi(\boldsymbol{R}) ; \delta \Psi(\boldsymbol{R} ; \delta \boldsymbol{R}))
$$

is zero for all $\delta \boldsymbol{R}$ if and only if $\delta \mathbb{J}_{P}(Q ; \delta Q)=\left\langle\delta Q, W-P Q^{-1}\right\rangle$ is zero for all $\delta Q$. Consequently, $\boldsymbol{J}_{P}$ has a stationary point at $\hat{\boldsymbol{R}}$ if and only if $\mathbb{J}_{P}$ has a stationary point at $\Psi(\hat{\boldsymbol{R}})$. However, $\mathbb{J}_{P}$ has exactly one such point, and hence the same holds for $\boldsymbol{J}_{P}$. Moreover, since $\delta^{2} \mathbb{J}_{P}(Q ; \delta Q)=\left\langle\delta Q, P Q^{-1} \delta Q Q^{-1}\right\rangle>0$ for all $\delta Q \neq 0$ and $\delta \rrbracket_{P}(\hat{Q} ; \delta Q)=0$ at the minimum $\hat{Q}$, the second directional derivative

$$
\delta^{2} \boldsymbol{J}_{P}(\hat{\boldsymbol{R}} ; \delta \boldsymbol{R})=\delta^{2} \mathbb{J}_{P}(\Psi(\hat{\boldsymbol{R}}) ; \delta \Psi(\hat{\boldsymbol{R}} ; \delta \boldsymbol{R}))+\delta \mathbb{J}_{P}\left(\Psi(\hat{\boldsymbol{R}}) ; \delta^{2} \Psi(\hat{\boldsymbol{R}} ; \delta \boldsymbol{R})\right)
$$

is positive for sufficiently small $\delta \boldsymbol{R} \neq 0$. Therefore, $\boldsymbol{J}_{P}$ is strictly convex in some neighborhood of $\hat{\boldsymbol{R}}$. 
6.2. The gradient and the Hessian of the new functional. In order to use Newton's method to solve the new optimization problem, we need to determine the gradient and the Hessian of $\boldsymbol{J}_{P}$. We begin with the gradient.

Proposition 6.2. Given the real $\ell \times \ell$ matrix-valued Fourier coefficients

$$
C_{k}=\frac{1}{2 \pi} \int_{-\pi}^{\pi} e^{i k \theta} P\left(e^{i \theta}\right)\left(R^{*}\left(e^{i \theta}\right) R\left(e^{i \theta}\right)\right)^{-1} d \theta, \quad k=0,1, \ldots, n
$$

and the modified Pick matrix $\boldsymbol{K}$, given by (6.5), the gradient of $\boldsymbol{J}_{P}$ is given by

$$
\frac{\partial \boldsymbol{J}_{P}}{\partial \boldsymbol{R}}(\boldsymbol{R})=2(\boldsymbol{K}-C(\boldsymbol{R})) \boldsymbol{R},
$$

where the $(n+1) \ell \times(n+1) \ell$ matrix $C(\boldsymbol{R})$ is the Toeplitz matrix

$$
C(\boldsymbol{R}):=\left[\begin{array}{cccc}
C_{0} & C_{1} & \cdots & C_{n} \\
C_{1}^{\top} & C_{0} & \ddots & \vdots \\
\vdots & \ddots & \ddots & C_{1} \\
C_{n}^{\top} & \cdots & C_{1}^{\top} & C_{0}
\end{array}\right] .
$$

The proof of Proposition 6.2 is given in Appendix B, while the proof of the following proposition, describing the Hessian of $\boldsymbol{J}_{P}$, is given in Appendix C.

Proposition 6.3. The Hessian of $\boldsymbol{J}_{P}$ is given by

$$
\frac{\partial^{2}}{(\partial \operatorname{vec} \boldsymbol{R})^{2}} \boldsymbol{J}_{P}(\boldsymbol{R})=2\left(I_{\ell} \otimes \boldsymbol{K}\right)-2 \frac{\partial^{2}}{(\partial \operatorname{vec} \boldsymbol{R})^{2}}\langle\log \operatorname{det} R, P\rangle \text {. }
$$

Here the component of the second term are obtained by rearranging the elements in

$$
\left(\frac{\partial}{\partial R_{j}} \otimes \frac{\partial}{\partial R_{k}}\right)\langle\log \operatorname{det} R, P\rangle=-S_{j+k}^{\top}, \quad j, k=0,1, \ldots, n,
$$

where $S_{0}, S_{1}, \ldots, S_{2 n}$ are defined via the expansion

$$
P(z)\left(\operatorname{vec} R(z)^{-1}\right)\left(\operatorname{vec} R(z)^{-\top}\right)^{\top}=\sum_{-\infty}^{\infty} S_{k} z^{-k} .
$$

Remark 6.4. Since the left hand side of (6.13) is the product of three factors, two of which have Laurent expansions with infinitely many terms, one might wonder how to determine the coefficients $S_{0}, S_{1}, \ldots, S_{2 n}$ in a finite number of operations. As we shall see in Appendix $\mathrm{C}$, this can be achieved by observing that $P(z)\left(R(z)^{\top} \otimes R(z)^{-1}\right)$ has the same elements as (6.13), appropriately rearranged, and can be factored as the product of two finite and one infinite Laurent expansion.

6.3. The central solution. The optimization problem to minimize $\boldsymbol{J}_{P}$ is particularly simple if $P=1$. In this case, and only in this case, the problem can be reduced to one of solving a system of linear equations. This solution is generally called the central solution. In fact, since $\operatorname{det} R(z)$ has no zeros in $\overline{\mathbb{D}}$, by the mean-value theorem of harmonic functions,

$$
\frac{1}{2 \pi} \int_{-\pi}^{\pi} \log \left|\operatorname{det} R\left(e^{i \theta}\right)\right| d \theta=\log |\operatorname{det} R(0)| .
$$


Consequently, since $\arg \operatorname{det} R\left(e^{-i \theta}\right)=-\arg \operatorname{det} R\left(e^{i \theta}\right)$,

$$
\boldsymbol{J}_{1}(\boldsymbol{R})=\operatorname{tr} \boldsymbol{R}^{\top} \boldsymbol{K} \boldsymbol{R}-2 \log \operatorname{det} R_{0} .
$$

Since det $R(z)$ has no zeros in the unit disc, $R_{0}$ is nonsingular. Therefore, setting the gradient of $\boldsymbol{J}_{1}(\boldsymbol{R})$ equal to zero, we obtain

$$
\boldsymbol{K} \boldsymbol{R}=\boldsymbol{E} R_{0}^{-\top}, \quad \boldsymbol{E}=\left[\begin{array}{llll}
I_{\ell} & 0 & \cdots & 0
\end{array}\right]^{\top},
$$

and therefore $R_{0}=\boldsymbol{E}^{\top} \boldsymbol{R}=\boldsymbol{E}^{\top} \boldsymbol{K}^{-1} \boldsymbol{E} R_{0}^{-\top}$, which yields

$$
R_{0} R_{0}^{\top}=\boldsymbol{E}^{\top} \boldsymbol{K}^{-1} \boldsymbol{E} .
$$

First solving (6.15) for the unique Cholesky factor and inserting into (6.14), (6.14) reduces to a linear system of equations that has a unique solution $\boldsymbol{R}$ since $\boldsymbol{K}$ is positive definite.

6.4. The continuation method. Now, we would like to find the minimizer of $\boldsymbol{J}_{P}$ for an arbitrary $P \in \mathcal{Q}_{+}(1, n)$. To this end, we construct a homotopy between the gradient of $\boldsymbol{J}_{1}$ and the gradient of $\boldsymbol{J}_{P}$ along the lines of [22,40], allowing us to pass from the central solution to the solution of interest.

Now, for any $\lambda \in[0,1]$, define

$$
P_{\lambda}(z):=1+\lambda(P(z)-1) .
$$

Then, since $Q_{+}(1, n)$ is convex, $P_{\lambda} \in Q_{+}(1, n)$. By Proposition 6.1, the functional

$$
\boldsymbol{J}_{P_{\lambda}}(\boldsymbol{R})=\operatorname{tr} \boldsymbol{R}^{\top} \boldsymbol{K} \boldsymbol{R}-2\left\langle\log \operatorname{det} R, P_{\lambda}\right\rangle
$$

has a unique minimum at $\hat{\boldsymbol{R}}(\lambda)$ and is locally strictly convex in some neighborhood of $\hat{\boldsymbol{R}}(\lambda)$. This point is the unique solution in $\mathcal{R}_{+}(\ell, n)$ of the nonlinear equation

$$
h(\boldsymbol{R}, \lambda):=\frac{\partial \boldsymbol{J}_{P_{\lambda}}(\boldsymbol{R})}{\partial \operatorname{vec} \boldsymbol{R}}=0 .
$$

Then the function $h: \mathcal{R}_{+}(\ell, n) \times[0,1] \rightarrow \mathbb{R}^{(n+1) \ell^{2}}$ is a homotopy from the gradient of $\boldsymbol{J}_{1}$ to the gradient of $\boldsymbol{J}_{P}$. In particular, $\hat{\boldsymbol{R}}(0)$ is the central solution.

In view of the strict local convexity of $\boldsymbol{J}_{P_{\lambda}}$ in a neighborhood of $\hat{\boldsymbol{R}}(\lambda)$, the Jacobian of $h(\boldsymbol{R}, \lambda)$ is positive definite at $\hat{\boldsymbol{R}}(\lambda)$. Consequently, by the implicit function theorem, the function $\lambda \rightarrow \hat{\boldsymbol{R}}(\lambda)$ is continuously differentiable on the interval $[0,1]$, and

$$
\frac{d}{d \lambda} \operatorname{vec} \hat{\boldsymbol{R}}(\lambda)=-\left.\left(\frac{\partial \boldsymbol{h}}{\partial \operatorname{vec} \boldsymbol{R}}(\boldsymbol{R}, \lambda)\right)^{-1}\left(\frac{\partial \boldsymbol{h}}{\partial \lambda}(\boldsymbol{R}, \lambda)\right)\right|_{\boldsymbol{R}=\hat{\boldsymbol{R}}_{(\lambda)}},
$$

where the inverted matrix is the Hessian of $\boldsymbol{J}_{P_{\lambda}}$ that can be determined as in Proposition 6.3. We want to follow the trajectory $\hat{\boldsymbol{R}}(\lambda)$ defined by the solution of this differential equation with the central solution as the initial condition.

To this end, we construct an increasing sequence of numbers $\lambda_{0}, \lambda_{1}, \ldots, \lambda_{N}$ on the interval $[0,1]$ with $\lambda_{0}=0$ and $\lambda_{N}=1$. Then, for $k=1,2, \ldots, N$, we solve the nonlinear equation $h\left(\boldsymbol{R}, \lambda_{k}\right)=0$ for vec $\hat{\boldsymbol{R}}\left(\lambda_{k}\right)$ by Newton's method with initial condition

$$
\operatorname{vec} \boldsymbol{R}_{0}\left(\lambda_{k}\right)=\operatorname{vec} \hat{\boldsymbol{R}}\left(\lambda_{k-1}\right)+\frac{d}{d \lambda} \operatorname{vec} \hat{\boldsymbol{R}}\left(\lambda_{k}\right)\left(\lambda_{k}-\lambda_{k-1}\right)
$$


The numbers $\lambda_{0}, \lambda_{1}, \ldots, \lambda_{N}$ have to be chosen close enough so that, for each $k=$ $1,2, \ldots, N, \boldsymbol{R}_{0}\left(\lambda_{k}\right)$ lies in the local convexity region of $\boldsymbol{J}_{P_{\lambda_{k}}}$, guaranteeing that Newton's method converges to $\hat{\boldsymbol{R}}\left(\lambda_{k}\right)$. Strategies for choosing $\lambda_{0}, \lambda_{1}, \ldots, \lambda_{N}$ are given in [40].

Remark 6.5. A MATLAB implementation of this algorithm is available [1].

\section{An APplication to A BENCHMARK PROBLEM IN ROBUST CONTROL}

During the last two decades it has been discovered that analytic interpolation theory is closely related to several robust control problems, for example, the gain-margin maximization problem [46, 47, 32], the robust stabilization problem [33], sensitivity shaping in feedback control, simultaneous stabilization [28], the robust regulation problem [15], the general $H^{\infty}$ control problem [23], and, more generally, the model matching problem. In this section we apply the theory of this paper to a benchmark problem in sensitivity shaping for a MIMO plant from a popular textbook on multivariable control by Maciejowski [37]. We refer the reader to Example 2.2 in Section 2 for notation.

The control system in [37] describes the vertical-plane dynamics of an airplane and can be linearized to yield a linear system $\mathrm{P}$ with three inputs, three outputs and five states, namely

$$
\begin{aligned}
& \dot{x}=A x+B u \\
& y=C x+D u,
\end{aligned}
$$

where

$$
\begin{aligned}
& A=\left[\begin{array}{rrrrr}
0 & 0 & 1.1320 & 0 & -1.000 \\
0 & -0.0538 & -0.1712 & 0 & 0.0705 \\
0 & 0 & 0 & 1.0000 & 0 \\
0 & 0.0485 & 0 & -0.8556 & -1.013 \\
0 & -0.2909 & 0 & 1.0532 & -0.6859
\end{array}\right] \quad C=\left[\begin{array}{lllll}
1 & 0 & 0 & 0 & 0 \\
0 & 1 & 0 & 0 & 0 \\
0 & 0 & 1 & 0 & 0
\end{array}\right] \\
& B=\left[\begin{array}{rrr}
0 & 0 & 0 \\
-0.12 & 1.0000 & 0 \\
0 & 0 & 0 \\
4.4190 & 0 & -1.665 \\
1.5750 & 0 & -0.0732
\end{array}\right]
\end{aligned}
$$

This system is not asymptotically stable due to the pole at the origin. It is strictly proper $(D=0)$ and the first Markov coefficient

$$
C B=\left[\begin{array}{ccc}
0 & 0 & 0 \\
-0.12 & 1 & 0 \\
0 & 0 & 0
\end{array}\right]
$$

is rank deficient.

We want to design a controller $\mathrm{C}$ as in Figure 2 in Section 2 that renders the closedloop system robust against various disturbances. More precisely, the specifications are

- Bandwidth about $10 \mathrm{rad} / \mathrm{s}$

- Integral action in each loop

- Well-damped step responses 
By exploiting the design freedom offered by choosing the design parameters, namely an upper limit $\gamma$ of the gain, the tuning parameters $\rho_{0}, \rho_{1}, \ldots, \rho_{n}$, and additional interpolation constraints, we shape the sensitivity function to meet the specifications, while limiting the degree of the controller.

First we deal with the pole at origin. By perturbing the $A$ matrix we move the pole into the right half-plane, generating an interpolation point as described in Section 2. More precisely, we move the pole to $10^{-6}$ by increasing $A_{11}$ to $10^{-6}$. This will ensure integral action in each loop by bringing the sensitivity to zero near zero frequency. Since the plant is strictly proper, we also add an interpolation point of multiplicity three at the very high frequency $10^{8}$, which forces the controller to be strictly proper and creates a steep "roll-off" of the complementary sensitivity function. Then the class of interpolants becomes

$$
\left\{S \in R H^{\infty}: \begin{array}{l}
S\left(10^{8}\right)=I, S^{\prime}\left(10^{8}\right)=S^{\prime \prime}\left(10^{8}\right)=0 \\
S\left(10^{-6}\right)=0,\|S\|_{\infty} \leq \gamma, \operatorname{deg} S \leq 9
\end{array}\right\}
$$

where $\gamma$ is a bound to be selected in the design. By means of a linear fractional transformation and an appropriate scaling, we transform the problem to the form considered in this paper, yielding the family

$$
\left\{F \in \mathcal{F}_{+}(3): F(0)=1.2222 I, F^{\prime}(0)=F^{\prime \prime}(0)=0, F(0.9995)=I\right\} .
$$

Next, we tune the design parameters to meet the design specs. First we pick the upper bound $\gamma=10$. However, the actual maximal norm of the sensitivity will be considerably smaller. Furthermore, we want to peak the sensitivity function slightly above $10 \mathrm{rad} / \mathrm{s}$. We can achieve this by choosing spectral zeros close to the imaginary axis in the corresponding region. Here, we first pick the points $\{12, \pm 18 i\}$ and transform them to the unit disc by the same linear fractional transformation as for the interpolation points. By rescaling each resulting point to have absolute value less than 0.8, if necessary, we avoid numerical difficulties and prevent the peak of $|S|$ from becoming too high. In this way, we obtain the spectral zeros $\{0.7320,0.7015 \pm 0.3845 i\}$, which we use in the algorithm of Section 6 to determine the corresponding unique interpolant $F$. Then we transform back to $S$ and calculate $C(s)=P(s)^{-1}\left(S(s)^{-1}-I\right)$.

In Table 1 we compare our control design with an optimal $H^{\infty}$ design using the weighting functions of [37]. In Figure 5 the (singular-value) frequency responses of the sensitivity and the complementary sensitivity of both designs are plotted, and in Figure 6 the step responses are depicted. Clearly, both designs meet the design specifications. We emphasize that although our design meets the specifications at least as well as does the $H^{\infty}$ design, the McMillan degree of our controller is only half of that of the $H^{\infty}$ controller.

\begin{tabular}{l||c|c} 
& $\begin{array}{c}\text { Method of } \\
\text { this paper }\end{array}$ & $\begin{array}{c}\text { Optimal } \\
H^{\infty} \text { design }\end{array}$ \\
\hline \hline Controller degree & 8 & 17 \\
Peak $\|S\|_{\infty}(\mathrm{dB})$ & 1.3849 & 1.3910 \\
Peak $\|T\|_{\infty}(\mathrm{dB})$ & 1.0000 & 1.2565 \\
Bandwidth $S(\mathrm{rad} / \mathrm{s})$ & 4.0050 & 3.7720 \\
Bandwidth $T(\mathrm{rad} / \mathrm{s})$ & 9.1825 & 10.6913
\end{tabular}

Table 1. Summary of the designs. 

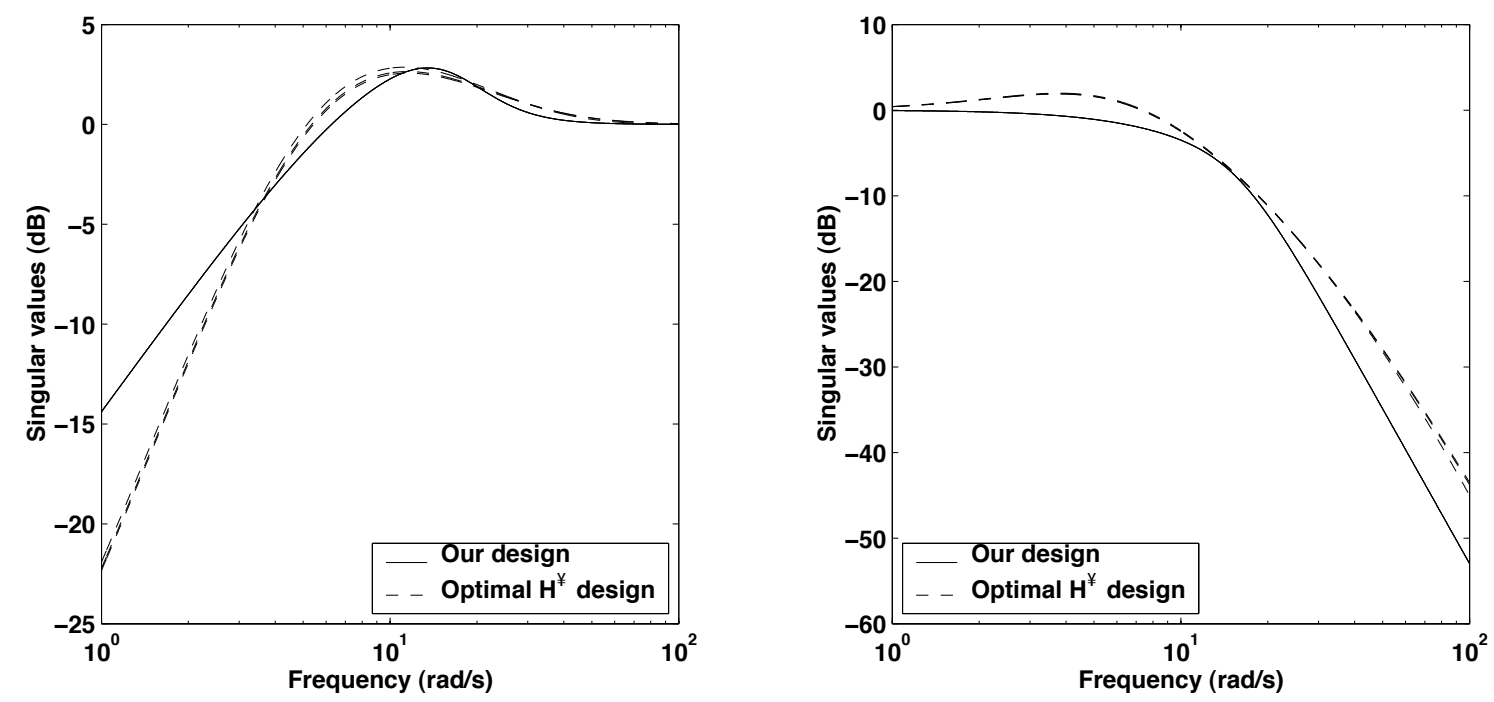

Figure 5: Singular value plots of the sensitivity and the complementary sensitivity.

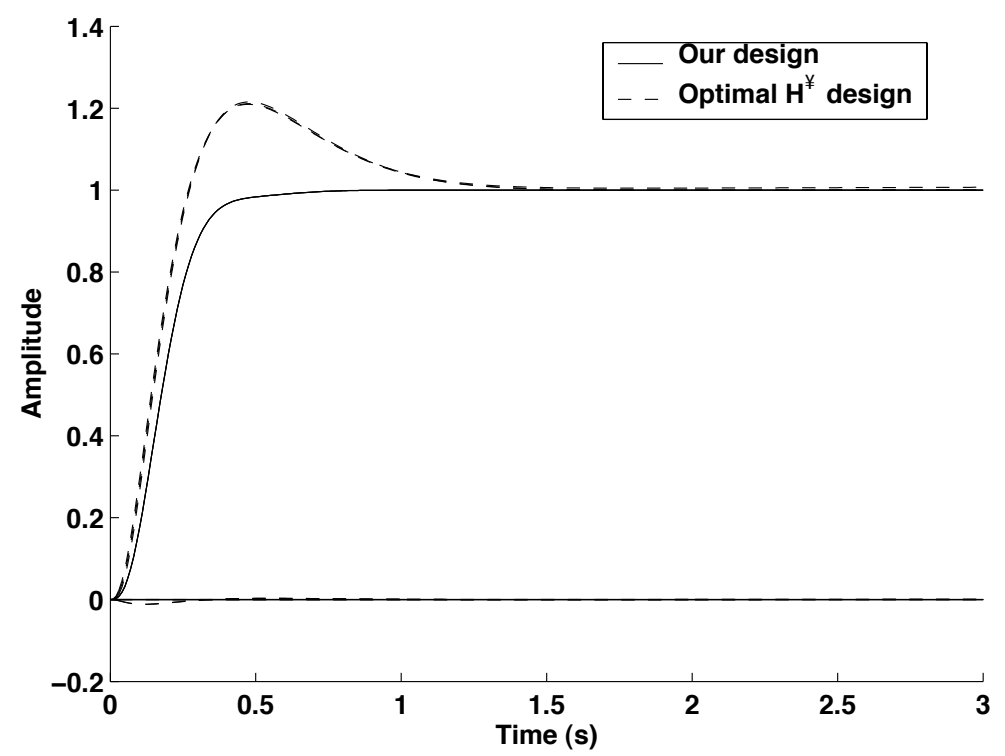

Figure 6: The step responses for the $H^{\infty}$-design and our design.

\section{Conclusions}

In this paper we have developed a theory for matrix-valued Nevanlinna-Pick interpolation with complexity constraint. We have shown that the spectral zeros characterize completely a class of interpolants of a bounded complexity. We have devised a numerically stable algorithm based on homotopy continuation to compute any such interpolants. The potential advantage of the theory and the algorithm was illustrated by a benchmark multivariable control example.

The standard $H^{\infty}$ control problem can be reduced to not only matrix-valued interpolation but also tangential interpolation. We expect that the reduction to the tangential Nevanlinna-Pick interpolation problem will be more natural in the sense that the degree bound can be much lower than the one in this paper (see $[34,35]$ ). 
Therefore, it will be important to modify our theory to tangential Nevanlinna-Pick interpolation. This is the subject of future study.

\section{Appendix A. Nonsingularity of the Jacobian MAtrix $\delta \Psi$}

To show that the Jacobian matrix of $\Psi$ in Proposition 6.1 is bijective, we prove a somewhat more general statement.

Lemma A.1. Let $\mathcal{V}(\ell, n)$ be the class of real $\ell \times \ell$ matrix polynomials

$$
V(z)=V_{0}+V_{1} z+\cdots+V_{n} z^{n}
$$

such that $V_{0}$ is upper triangular, and let $R \in \mathcal{V}(\ell, n)$ have the properties that the constant term $R_{0}$ is nonsingular and that $\operatorname{det} R$ and $\operatorname{det} R^{*}$ have no roots in common. Then, the linear map $S(R)$ sending $V \in \mathcal{V}(\ell, n)$ to

$$
S(R) V:=R(z) V^{*}(z)+V(z) R^{*}(z)
$$

is nonsingular.

Proof. Since $S(R)$ is a linear map between Euclidean spaces of the same dimension, it suffices to prove that $S(R)$ is injective. Without restriction we may assume that $R(z)$ is upper triangular. In fact, let $T(z)$ be a unimodular matrix polynomial with $T(0)$ upper triangular such that $T(z) R(z)$ is upper triangular. Such a $T$ indeed exists due to the procedure deriving the Smith form [24]. Then

$$
T S(R) V T^{*}=(T R)(T V)^{*}+(T V)(T R)^{*}=0
$$

if and only if $S(R) V=0$. Moreover, the new $V_{0}$, i.e., $T(0) V(0)$, is still upper triangular. In this formulation

$$
\operatorname{det} R(z)=r_{11}(z) r_{22}(z) \cdots r_{\ell \ell}(z)
$$

where $r_{11}, r_{22}, \cdots, r_{\ell \ell}$ are the diagonal elements in $R$. In particular, by assumption, no $r_{i i}$ can have zeros in common with any $r_{j j}^{*}$. It then remains to prove that

$$
R V^{*}+V R^{*}=0
$$

implies $V=0$.

The proof is by induction. The statement clearly holds for $\ell=1$. In fact, if $R\left(z_{j}\right)=$ 0 , then, by assumption, $R^{*}\left(z_{j}\right) \neq 0$, and hence, by (A.1), $V\left(z_{j}\right)=0$. Consequently, we must have $V(z)=\lambda(z) R(z)$ for some real polynomial $\lambda$, which inserted into (A.1) yields

$$
\left(\lambda+\lambda^{*}\right) R R^{*}=0
$$

This implies that $\lambda=0$ and hence that $V=0$, as claimed. 
Now, suppose that (A.1) implies $V=0$ for $\ell=k-1$. Then, for $\ell=k,($ A.1) can be written

$$
\begin{aligned}
{\left[\begin{array}{c|ccc}
r_{11} & r_{12} & \cdots & r_{1 k} \\
\hline 0 & & & \\
\vdots & & \hat{R} & \\
0 & & &
\end{array}\right] } & {\left[\begin{array}{c|ccc}
v_{11}^{*} & v_{21}^{*} & \cdots & v_{k 1}^{*} \\
\hline v_{12}^{*} & & & \\
\vdots & & \hat{V}^{*} & \\
v_{1 k}^{*} & & &
\end{array}\right] } \\
+ & +\left[\begin{array}{c|ccc}
v_{11} & v_{12} & \cdots & v_{1 k} \\
\hline v_{21} & & & \\
\vdots & & \hat{V} & \\
v_{k 1} & & &
\end{array}\right]\left[\begin{array}{c|cccc}
r_{11}^{*} & 0 & \cdots & 0 & 0 \\
\hline r_{12}^{*} & & & \\
\vdots & & & \hat{R}^{*} & \\
r_{1 k}^{*} & & &
\end{array}\right]=0,
\end{aligned}
$$

which, in particular, contains the $(k-1) \times(k-1)$ matrix relation $\hat{R} \hat{V}^{*}+\hat{V} \hat{R}^{*}=0$ of type (A.1). Consequently, by the induction assumption, $\hat{V}=0$, so, to prove that $V=0$, it just remain to show that the border elements $v_{11}, v_{12}, \ldots, v_{1 k}, v_{21}, \ldots, v_{k 1}$ are all zero. To this end, let us begin with the corner elements $v_{1 k}$ and $v_{k 1}$. From the $(1, k)$ and $(k, 1)$ elements in (A.2), we have

$$
\begin{aligned}
& r_{11} v_{k 1}^{*}+v_{1 k} r_{k k}^{*}=0 \\
& v_{1 k}^{*} r_{k k}+v_{k 1} r_{11}^{*}=0 .
\end{aligned}
$$

In the same way as in the case $\ell=1$, (A.3) implies that $v_{1 k}=\lambda_{1 k} r_{11}$ for some real polynomial $\lambda_{1 k}$, and (A.4) implies that $v_{k 1}=\lambda_{k 1} r_{k k}$ for some real polynomial $\lambda_{k 1}$, which inserted into (A.3) yields

$$
\left(\lambda_{k 1}+\lambda_{1 k}^{*}\right) r_{11} r_{k k}^{*}=0 .
$$

This implies that $\lambda_{k 1}$ and $\lambda_{1 k}$ are real numbers such that $\lambda_{1 k}=-\lambda_{k 1}$. However, by assumption, $V(0)$ is upper triangular, and $R(0)$ is upper triangular and nonsingular. Hence $v_{k 1}(0)=0$ and $r_{k k}(0) \neq 0$, implying that $\lambda_{k 1}=v_{k 1}(0) / r_{k k}(0)=0$ and, consequently, $\lambda_{1 k}=0$. Since, therefore, $v_{1 k}=0$ and $v_{k 1}=0$, (A.2) now takes the form

$$
\left[\begin{array}{c|c}
\tilde{R} & * \\
\hline 0 & *
\end{array}\right]\left[\begin{array}{c|c}
\tilde{V}^{*} & 0 \\
\hline 0 & 0
\end{array}\right]+\left[\begin{array}{c|c}
\tilde{V} & 0 \\
\hline 0 & 0
\end{array}\right]\left[\begin{array}{c|c}
\tilde{R}^{*} & 0 \\
\hline * & *
\end{array}\right]=0,
$$

which only yields the $(k-1) \times(k-1)$ matrix relation $\tilde{R} \tilde{V}^{*}+\tilde{V} \tilde{R}^{*}=0$ of type (A.1). However, by the induction assumption, $\tilde{V}=0$. Therefore, $V=0$ in the case $\ell=k$ also, so, by induction, $V=0$ for all $k$.

\section{Appendix B. Computing the Gradient}

To establish the expression (6.9) in Proposition 6.2 for the gradient

$$
\frac{\partial \boldsymbol{J}_{P}}{\partial \boldsymbol{R}}(\boldsymbol{R})=2\left(\boldsymbol{K} \boldsymbol{R}-\frac{\partial}{\partial \boldsymbol{R}}\langle\log \operatorname{det} R, P\rangle\right)
$$


of (6.7), we need to determine

$$
\begin{aligned}
& \frac{\partial}{\partial R_{k}}\langle\log \operatorname{det} R, P\rangle=\frac{1}{2 \pi} \int_{-\pi}^{\pi} \frac{\partial}{\partial R_{k}} \log \operatorname{det} R^{*}\left(e^{i \theta}\right) P\left(e^{i \theta}\right) d \theta \\
& =\frac{1}{2 \pi} \int_{-\pi}^{\pi} e^{-i k \theta} R^{*}\left(e^{i \theta}\right)^{-\mathrm{T}} P\left(e^{i \theta}\right) d \theta \\
& =\frac{1}{2 \pi} \int_{-\pi}^{\pi} P\left(e^{i \theta}\right)\left(R^{*}\left(e^{i \theta}\right) R\left(e^{i \theta}\right)\right)^{-\top} R^{\top}\left(e^{i \theta}\right) e^{-i k \theta} d \theta \\
& =\left[\begin{array}{lllll}
C_{k}^{\top} & \cdots & C_{0} & \cdots & C_{n-k}
\end{array}\right] \boldsymbol{R}
\end{aligned}
$$

where $C_{k}$ is defined by (6.8). This completes the proof of Proposition 6.2.

Next, we explain how to actually compute $C_{0}, C_{1}, \ldots, C_{n}$. In view of (4.13),

$$
P\left(R^{*} R\right)^{-1}=\rho \rho^{*}\left[(\tau R)^{*}(\tau R)\right]^{-1} .
$$

We can determine $\hat{C}_{0}, \hat{C}_{1}, \ldots, \hat{C}_{2 n}$ in the expansion

$$
\left[(\tau R)^{*}(\tau R)\right]^{-1}=\hat{C}_{0}+\hat{C}_{1} z+\hat{C}_{1}^{\top} z^{-1}+\cdots+\hat{C}_{2 n} z^{2 n}+\hat{C}_{2 n}^{\top} z^{-2 n}+\cdots .
$$

by solving a system of linear equations. Now, defining

$$
\mu(z):=\mu_{0}+\sum_{s=1}^{n} \mu_{\ell}\left(z^{s}+z^{-s}\right)=\rho(z) \rho^{*}(z),
$$

we can identify matrix coefficients of equal powers in $z$ in

$$
\mu\left[(\tau R)^{*}(\tau R)\right]^{-1}=C_{0}+C_{1} z+C_{1}^{\top} z^{-1}+\cdots+C_{n} z^{n}+C_{n}^{\top} z^{-n}+\cdots,
$$

to obtain

$$
\left[\begin{array}{c}
C_{0} \\
C_{1} \\
\vdots \\
C_{n}
\end{array}\right]=\left(\left[\begin{array}{cccc}
\hat{C}_{0} & \hat{C}_{1} & \cdots & \hat{C}_{n} \\
\hat{C}_{1} & \hat{C}_{0} & \cdots & \hat{C}_{n-1} \\
\vdots & \vdots & \ddots & \vdots \\
\hat{C}_{n} & \hat{C}_{n-1} & \cdots & \hat{C}_{0}
\end{array}\right]+\left[\begin{array}{cccc}
\hat{C}_{0} & \hat{C}_{1} & \cdots & \hat{C}_{n} \\
\hat{C}_{1} & \hat{C}_{2} & \cdots & \hat{C}_{n+1} \\
\vdots & \vdots & \cdot & \vdots \\
\hat{C}_{n} & \hat{C}_{n+1} & \cdots & \hat{C}_{2 n}
\end{array}\right]\right)\left[\begin{array}{c}
\mu_{0} I / 2 \\
\mu_{1} I \\
\vdots \\
\mu_{n} I
\end{array}\right]
$$

\section{Appendix C. Computing the Hessian}

We begin by proving Proposition 6.3. Since

$$
\frac{\partial^{2}\left(\operatorname{tr} \boldsymbol{R}^{\top} \boldsymbol{K} \boldsymbol{R}\right)}{\partial \operatorname{vec} \boldsymbol{R}^{2}}=2\left(I_{\ell} \otimes \boldsymbol{K}\right),
$$

it remains to establish (6.12). Since

$$
\frac{\partial}{\partial R_{j}} \otimes \frac{\partial}{\partial R_{k}}\langle\log \operatorname{det} R, P\rangle=\frac{1}{2 \pi} \int_{-\pi}^{\pi} \frac{\partial}{\partial R_{j}} \otimes R^{*}\left(e^{i \theta}\right)^{-\top} P\left(e^{i \theta}\right) e^{-i k \theta} d \theta,
$$

(6.12) would follow if we could show that

$$
\frac{\partial}{\partial R_{j}} \otimes R^{*}(z)^{-\top}=-z^{-j} \operatorname{vec}\left(R^{*}\right)^{-\top}\left(\operatorname{vec} R^{-*}\right)^{\top} .
$$


Since $R^{-*}(z)^{\top} R^{*}(z)^{\top} \equiv I$, denoting the $(s, t)$ element of $R_{j}$ by $R_{j}^{s t}$ we obtain

$$
\frac{\partial}{\partial R_{j}^{s t}} R^{*}(z)^{-\top}=-\left(R^{*}\right)^{-\top} \frac{\partial\left(R^{*}\right)^{\top}}{\partial R_{j}^{s t}}\left(R^{*}\right)^{-\top}=-z^{-j}\left(R^{*}\right)^{-\top} e_{s} e_{t}^{\top}\left(R^{*}\right)^{-\top},
$$

and therefore

$$
\begin{aligned}
\frac{\partial}{\partial R_{j}} \otimes R^{*}(z)^{-\top} & :=\left[\begin{array}{ccc}
\frac{\partial}{\partial R_{j}^{11}} R^{*}(z)^{-\mathrm{T}} & \cdots & \frac{\partial}{\partial R_{j}^{1 \ell}} R^{*}(z)^{-\mathrm{T}} \\
\vdots & \ddots & \vdots \\
\frac{\partial}{\partial R_{j}^{\ell 1}} R^{*}(z)^{-\top} & \cdots & \frac{\partial}{\partial R_{j}^{\ell \ell}} R^{*}(z)^{-\mathrm{\top}}
\end{array}\right] \\
& =-z^{-j} \operatorname{vec}\left(R^{*}\right)^{-\mathrm{T}}\left(\operatorname{vec} R^{-*}\right)^{\top},
\end{aligned}
$$

establishing (C.1), and hence proving Proposition 6.3.

Next, we answer the question in Remark 6.4. To compute $S_{0}, S_{1}, \ldots, S_{2 n}$, we first expand

$$
P(z)\left(R^{-\top}(z) \otimes R^{-1}(z)\right)=\cdots+\tilde{S}_{2 n} z^{-2 n}+\cdots+\tilde{S}_{1} z^{-1}+\tilde{S}_{0}+\cdots,
$$

and transform $\tilde{S}_{k}$ to the coefficient matrices of $P\left(\operatorname{vec} R^{-1}\right)\left(\operatorname{vec} R^{-\top}\right)^{\top}$ by comparing the elements of $R^{-\top} \otimes R^{-1}$ with those of $\left(\operatorname{vec} R^{-1}\right)\left(\operatorname{vec} R^{-\top}\right)^{\top}$. The computation of $\tilde{S}_{k}$ can be done by first observing that

$$
\begin{aligned}
R^{-\top} \otimes R^{-1} & =\left(R^{*}\right)^{\top}\left(R^{-*}\right)^{\top} R^{-\top} \otimes\left(R^{*} R^{-*} R^{-1}\right) \\
& =\left(R^{*}\right)^{\top}\left(R^{*} R\right)^{-\top} \otimes\left(R^{*}\left(R R^{*}\right)^{-1}\right) \\
& =\left(\left(R^{*}\right)^{\top} \otimes R^{*}\right)\left(\left(R^{*} R\right)^{-\top} \otimes\left(R R^{*}\right)^{-1}\right) \\
& =\left(\left(R^{*}\right)^{\top} \otimes R^{*}\right)\left(\left(R^{*} R\right)^{\top} \otimes\left(R R^{*}\right)\right)^{-1} \\
& =\left(\left(R^{*}\right)^{\top} \otimes R^{*}\right)\left(\left(R^{\top} \otimes R\right)\left(\left(R^{*}\right)^{\top} \otimes R^{*}\right)\right)^{-1} \\
& =\left(\left(R^{*}\right)^{\top} \otimes R^{*}\right)\left(\left(R^{\top} \otimes R\right)\left(R^{\top} \otimes R\right)^{*}\right)^{-1},
\end{aligned}
$$

where we have used properties of the Kronecker product that may be found in, e.g., [29]. Multiplying this by $P$ then yields

$$
\begin{aligned}
P\left(R^{-\top} \otimes R^{-1}\right)= & \mu\left(\left(R^{*}\right)^{\top} \otimes R^{*}\right)\left(\left(\tau R^{\top} \otimes R\right)\left(\tau R^{\top} \otimes R\right)^{*}\right)^{-1} \\
= & \underbrace{\left(\mu_{0}+\mu_{1}\left(z+z^{-1}\right)+\cdots+\mu_{n}\left(z^{n}+z^{-n}\right)\right)}_{\mu} \\
& \times \underbrace{\left(U_{0}+U_{1} z^{-1}+\cdots+U_{2 n} z^{-2 n}\right)}_{\left(R^{*}\right)^{\top} \otimes R^{*}} \underbrace{\left(T_{0}+T_{1} z+T_{1}^{\top} z^{-1}+\cdots\right)}_{\left(\left(\tau R^{\top} \otimes R\right)\left(\tau R^{\top} \otimes R\right)^{*}\right)^{-1}},
\end{aligned}
$$

from which we can compute $\tilde{S}_{k}$.

\section{REFERENCES}

[1] The matlab codes are available at http://www.math.kth.se/ ${ }^{2}$ andersb/software.html.

[2] A. Blomqvist and R. Nagamune. An extension of a Nevanlinna-Pick interpolation solver to cases including derivative constraints. Preprint.

[3] R. W. Brockett. Finite Dimensional Linear Systems. John Wiley \& Sons, New York, 1970.

[4] C. I. Byrnes, P. Enqvist, and A. Lindquist. Cepstral coefficients, covariance lags and pole-zero models for finite data strings. IEEE Trans. Signal Processing, 49(4):677-693, April 2001. 
[5] C. I. Byrnes, P. Enqvist, and A. Lindquist. Identifiability and well-posedness of shaping-filter parameterizations: A global analysis approach. SIAM J. Contr. and Optimiz., 41(1):23-59, 2002.

[6] C. I. Byrnes, T. T. Georgiou, and A. Lindquist. A New Approach to Spectral Estimation: A Tunable High-Resolution Spectral Estimator. IEEE Trans. Signal Processing, 48(11):3189-3205, November 2000.

[7] C. I. Byrnes, T. T. Georgiou, and A. Lindquist. A generalized entropy criterion for NevanlinnaPick interpolation with degree constraint. IEEE Trans. Automat. Control, 46(6):822-839, June 2001.

[8] C. I. Byrnes, S. V. Gusev, and A. Lindquist. A convex optimization approach to the rational covariance extension problem. SIAM J. Contr. and Optimiz., 37(1):211-229, 1998.

[9] C. I. Byrnes, S. V. Gusev, and A. Lindquist. From finite covariance windows to modeling filters: A convex optimization approach. SIAM Review, 43(4):645-675, 2001.

[10] C. I. Byrnes and A. Lindquist. A convex optimization approach to generalized moment problems. Chapter in book to be published by Birkhäuser.

[11] C. I. Byrnes and A. Lindquist. Interior point solutions of variational problems and global inverse function theorems. Preprint.

[12] C. I. Byrnes and A. Lindquist. On the duality between filtering and Nevanlinna-Pick interpolation. SIAM J. Contr. and Optimiz., 39(3):757-775, 2000.

[13] C. I. Byrnes, A. Lindquist, S. V. Gusev, and A. S. Matveev. A Complete Parameterization of All Positive Rational Extensions of a Covariance Sequence. IEEE Trans. Automat. Control, 40(11):1841-1857, November 1995.

[14] P. E. Caines. Linear Stochastic Systems. John Wiley \& Sons, New York, 1988.

[15] M. K. K. Cevik and J. M. Schumacher. The Robust Regulation Problem with Robust Stability. Technical Report MAS-R9827, CWI, Amsterdam, 1999.

[16] B.-C. Chang and J. B. Pearson. Optimal Disturbance Reduction in Linear Multivariable Systems. IEEE Trans. Automat. Control, 29(10):880-887, October 1984.

[17] E. J. Davison, editor. Benchmark problems for control system design, May 1990. Report of the IFAC theory committee.

[18] Ph. Delsarte, Y. Genin, and Y. Kamp. Orthogonal Polynomial Matrices on the Unit Circle. IEEE Trans. Circuits and Systems, 25(3):149-160, March 1978.

[19] Ph. Delsarte, Y. Genin, and Y. Kamp. The Nevanlinna-Pick Problem for Matrix-valued Functions. SIAM J. Appl. and Math., 36:47-61, Feb 1979.

[20] Ph. Delsarte, Y. Genin, and Y. Kamp. Schur Parametrization of Positive Definite Block-Toeplitz Systems. SIAM J. Appl. and Math., 36:34-46, Feb 1979.

[21] J. C. Doyle, B. A. Francis, and A. R. Tannenbaum. Feedback Control Theory. Macmillan Publishing Company, New York, 1992.

[22] P. Enqvist. A homotopy approach to rational covariance extension with degree constraint. Int. J. Applied Mathematics and Computer Science, 11(5):1173-1201, 2001.

[23] B. A. Francis. A Course in $H_{\infty}$ Control Theory. Lecture Notes in Control and Information Sciences. Springer-Verlag, 1987.

[24] F. R. Gantmacher. The Theory of Matrices. Chelsea, New York, 1959.

[25] T. T. Georgiou. Realization of power spectra from partial covariance sequences. IEEE Trans. Acoustics, Speech and Signal Processing, 35:438-449, 1987.

[26] T. T. Georgiou. A Topological Approach to Nevanlinna-Pick Interpolation. SIAM J. Math. and Anal., 18(5):1248-1260, 1987.

[27] T. T. Georgiou. The Interplation Problem with a Degree Constraint. IEEE Trans. Automat. Control, 44(3):631-635, March 1999.

[28] B. K. Ghosh. Transcendental and interpolation methods in simultaneous stabilization and simultaneous partial pole placement problems. SIAM J. Contr. and Optimiz., 24:1091-1109, 1986.

[29] A. Graham. Kronecker products and matrix calculus with applications. John Wiley \& Sons, 1981.

[30] J. Hadamard. Sur les correspondances ponctuelles. In Oeuvres, Editions du Centre Nationale de la Researche Scientifique, pages 383-384. Paris, 1968. 
[31] R. E. Kalman. Realization of covariance sequences. In Proc. Toeplitz Memorial Conference, Tel Aviv, Israel, 1981.

[32] P. P. Khargonekar and A. Tannenbaum. Non-Euclidian Metrics and the Robust Stabilization of Systems with Parameter Uncertainty. IEEE Trans. Automat. Control, 30(10):1005-1013, October 1985.

[33] H. Kimura. Robust stabilizability for a class of transfer functions. IEEE Trans. Automat. Control, 29(10):788-793, October 1984.

[34] H. Kimura. Conjugation, interpolation and model-matching in $H^{\infty}$. Int. J. Control, 49:269-307, 1989.

[35] D. J. N. Limebeer and B. D. O. Anderson. An Interpolation Theory Approach to $H^{\infty}$ Controller Degree Bounds. Linear Algebra and Its Application, 98:347-386, 1988.

[36] A. Lindquist. A new algorithm for optimal filtering of discrete-time stationary processes. SIAM J. Control, 12(4):736-746, 1974.

[37] J. M. Maciejowski. Multivariable Feedback Design. Addison-Wesley, Wokingham U.K., 1989.

[38] R. Nagamune. All controllers to robust regulation with robust stability and performance enhancement with a degree bound. Preprint.

[39] R. Nagamune. Closed-loop shaping based on the Nevanlinna-Pick interpolation with a degree bound. submitted to IEEE Trans. Automatic Control.

[40] R. Nagamune. A robust solver using a continuation method for nevanlinna-pick interpolation with degree constraint. submitted to IEEE Trans. Automatic Control.

[41] R. Nagamune. Sensitivity reduction for SISO systems using the Nevanlinna-Pick interpolation with degree constraint. In Proceedings of 14th International Symposium of Mathematical Theory of Networks and Systems, Perpignan, France, 2000.

[42] R. Nagamune and A. Lindquist. Sensitivity shaping in feedback control and analytic interpolation theory. In J.L. Medaldi et al, editor, Optimal Control and Partial Differential Equations, pages 404-413. IOS Press, Amsterdam, 2001.

[43] A. V. Oppenheim and R. W. Shafer. Digital Signal Processing. Prentice Hall, London, 1975.

[44] M. M. Seron, J. H. Braslavsky, and G. C. Goodwin. Fundamental Limitations in Filtering and Control. Springer-Verlag, 1997.

[45] T. Söderström and P. Stoica. System Identification. Prentice Hall, 1989.

[46] A. Tannenbaum. Feedback stabilization of linear dynamical plants with uncertainty in the gain factor. Int. J. Control, 32(1):1-16, 1980.

[47] A. Tannenbaum. Modified Nevanlinna-Pick interpolation and feedback stabilization of linear plants with uncertainty in the gain factor. Int. J. Control, 36(2):331-336, 1982.

[48] P. Whittle. On the fitting of multivariate autoregressions, and the approximate canonical factorization of a spectral density matrix. Biometrika, 50:129-134, 1963.

[49] R. A. Wiggins and E. A. Robinson. Recursive solution to the multichannel filtering problem. Journal Geophysical Research, 70:1885-1891, 1966.

[50] D. C. Youla and M. Saito. Interpolation with positive-real functions. J. Franlkin Institute, 284:77-108, 1967.

[51] K. Zhou. Essentials of Robust Control. Prentice-Hall, New Jersey, 1998. 